\title{
Higgs Detection via Decays to Leptons with the SDC Detector *
}

\author{
R. Michael Barnett, Kevin Einsweiler and Ian Hinchliffe
}

\author{
Theoretical Physics Group \\ Physics Divisiont \\ Lawrence Berkeley Laboratory \\ 1 Cyclotron Road \\ Berkeley, California 94720
}

\begin{abstract}
This document discusses the potential for the SDC detector to discover the Standard Model Higgs boson via its decays to 4 charged leptons or to 2 charged leptons and 2 neutrinos.
\end{abstract}

-This work was supported by the Direclor, Oflice of Energy Research, Office of Iligh Energy and Nuclear Physics, Division of Ifigh Fiergy Plyysics of LIe U.S. Departinent of Energy under Contract DF-AC03-76SP00098. 


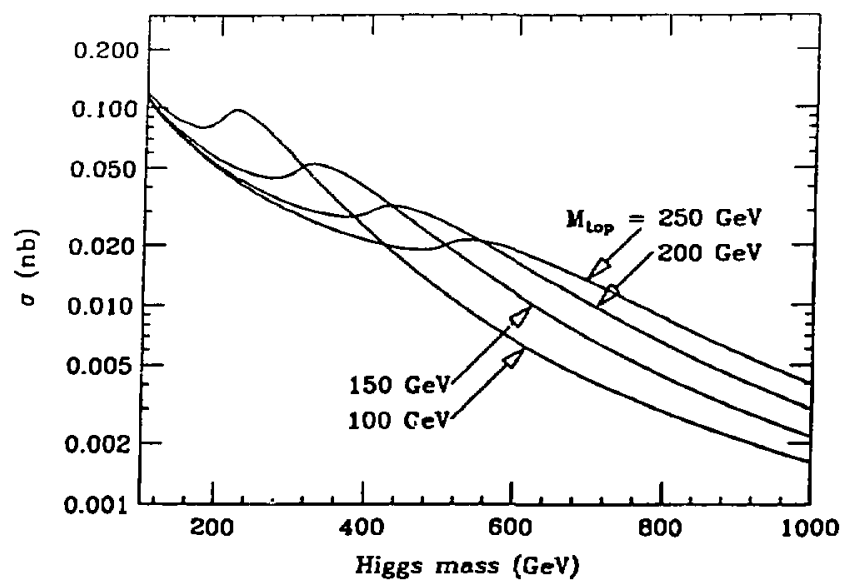

Figure 1: The cross section for the production of a Higgs boson in pp collisious at $\sqrt{s}=40 \mathrm{TeV}$ as a function of the Higgs boson mass. The lines are labelled by values of the top quark mass.

\section{Introduction}

Two mechanisms are relevant to the production of Higgs Bosons in hadron-hadron collisions; the process $g g \rightarrow H$ which proceeds via a virtual quark loop [1] and the process $q q \rightarrow q q H$ via the exchange of two $\mathrm{W}$ or $\mathrm{Z}$ bosons [2]. Since the coupling of the Higgs to a quark is proportional to the quark mass, the production rate from the first process is dependent on the top quark mass. Figure 1 shows the Higgs cross section that results from these processes at the SSC. It can be seen from this figure that the current allowed range of top quark masses betweed the lower limit of $89 \mathrm{GeV}$ from CDF [3] and the upper limit of $200 \mathrm{GeV}$ inferred from precision tests of the standard model [4] produces an uncertainty of approximately a factor of 4 in the Higgs production cross section. In the remainder of this work we shall use a top quark mass of $150 \mathrm{GeV}$.

We shall consider the decay of a Higgs to $\ell^{+} \ell^{-} \ell^{+} \ell^{-}$or $\ell^{+} \ell^{-} \nu \bar{\nu}$, where $\ell$ indicates either $e$ or $\mu$. These leptons will be required to pass isolation cuts, requiring that there be a limited amount of additional energy observed in a cone of radius $R=\sqrt{(\Delta \eta)^{2}+(\Delta \phi)^{2}}$ around the lipeon direction. Matching of calorimeter energies $(E)$ and tracking momenta $(p)$ is assumed to be pait of the electron identification process rather than part of the 
isolation criteria - the energy in the cone is computed from calorimeter measurements only.

Leptons arising from the Higgs decay have additional energy from the particles in the event that are produced at small $p_{t}$. The mean transverse momentum of particles in minimum bias events is expected to be $\sim 600 \mathrm{MeV}$ obtained by extrapolation from CDF[5] and UAl [6] which yields $\left\langle p_{\imath}\right\rangle=202+35 \log (\sqrt{s} / G e V)$ in $\mathrm{MeV}$. The energy from these particles is of order $0.5 \mathrm{GeV}$ per unit area in $\eta-\phi$ space, assuming that theie is only one event per beam crossing and that $d N / d \eta=5.5$. Events with a hard parton scattering such as those respossible for Higgs production can be expected to have more low $p_{t}$ particles arising from gluon radiation off the initial state partons. The total contribution of this underlying event to the energy in the $R$ cone is difficult to model in Monte-Carlos [7], but, in Higgs events, can be expected to be about $1 \mathrm{GeV}$ per unit area.

The transverse momentum of the leptons from Higgs decay is of order $M_{H} / 4$. Leptons of such high $p_{t}$ arising from the semileptonic decay of bottom and charm quarks will have energy close to them arising from the other fragments of the decaying quark itself as well as from the underlying event. Hence these leptons will be significantly less isolated than those from Higgs decay and the isolation requirement will be very important in rejecting possible backgrounds due to such heavy quark decays.

If the Higgs has mass larger than $2 M_{Z}$ then the relevant decays proceed via the decay $H \rightarrow Z Z$ and each $\ell^{+} \ell^{-}$pair will have is rariant mass $M_{Z}$. In this case the branching ratio to the $4 \ell(2 \ell \nu \nu)$ state is $0.00145(0.0084) .{ }^{2}$ If the Higgs is lighter than $2 M_{Z}$ the relevant decay is to one real $Z$ and the other virtual $\left(Z^{-}\right)$. In this latter case the branching ratio to $\ell^{+} \ell^{-} \ell^{+} \ell^{-}$falls rapidly as the mass decreases as is shown in Figure 2. In making this figure we have taken into account the QCD corrections [8] which act to reduce the decay width for $H \rightarrow b 5$ and hence increase the branching ratio to $2 Z^{*}$. This figure shows that the branching ratio to $Z Z^{*}$ bas a dip near $M_{H}=2 M_{W}$ where the decay channel $H \rightarrow W W$ opens. The branching ratio falls rapidly as the Higgs mass is reduced below $140 \mathrm{GeV}$. We will show that Higgs masses below $125 \mathrm{GeV}$ are not accessible unless the integrated luminosity substantially exceeds $10^{40} \mathrm{~cm}^{-2}$.

\section{Physics Simulation}

The figures in this document were generated using the PYTHIA [9] Monte-Carlo event generator (version 5.4) and the partonic generator PAPAGENO (version 3.30). The latter

\footnotetext{
'The lliggs decays dominantly to $W W$ and $Z Z$ fina states with relative rates or 2:1. The decay to $\vec{i}$ is never sufficiently large to reduce these rates by more than $10 \%$
} 


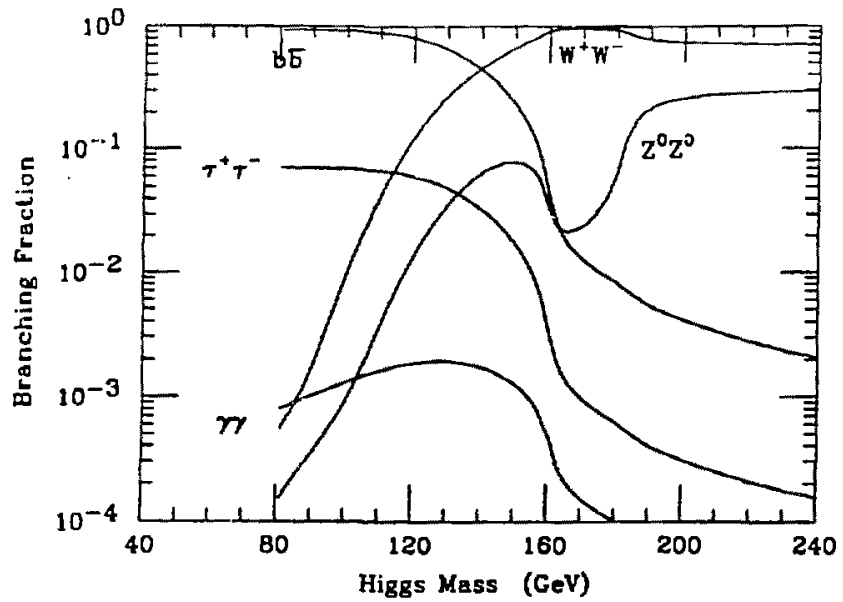

Figure 2: The branching ratio for a Higgs boson into various channels as a function of its mass.

is faster but the former must be used in discussing the details of, for example, the effect of isolation on lepton efficiencies and background rejections.

PY'THIA supplies many possible options for Higgs generation, and a brief description of what was done is supplied bere, in order to facilitate comparisons. There are four processes supported for Higgs production : $f \mathbf{f} \rightarrow H$ (Process 3 ), $q q Z Z \rightarrow q q H$ (Process 5), gqli' $1 \mathrm{l}^{\circ} \rightarrow q g H$ (Process 8 ), and $g g \rightarrow H$ (Process 102). The process $f \vec{f} \rightarrow H$ is ignored, as it is an approximation to the process $g g \rightarrow H+t \bar{t}$. The actual contribution from this process is small and the approximation is an overestimate, $s o$ it is more accurate to ignore it. Note that in all calculations made by this version of PYTHIA, the $Z$ 's are allowed to be off-shell as demanded by the available phase space, so $Z Z^{*}$ or even $Z^{*} Z^{*}$ production will automatically be generated. It should be further noted that Processes 5 and 8 use the s-pole approximation for the Higgs (although an energy-dependent Breit-Wigner is used for the line shape), which is known to overestimate the very high mass tail of a beavy Higgs. It is claimed that PYTHIA overestimates this rate by about $10 \%$ for $M_{Z Z}<1200$ $\mathrm{GeV}$ with an $800 \mathrm{GeV}$ Higgs mass. The $Z Z$ continuum background is generated using the PYTHIA $f \bar{f} \rightarrow Z Z$ contribution only (Process 22). This process contains the full $Z / \gamma$ propagator, and hence also contains the process $Z_{\gamma^{*}}$ in the approximation that the $\gamma^{-}$ is radiaied from a quark line (this is important for the $H \rightarrow Z Z^{-}$background analysis). 


\begin{tabular}{|c|cc|ccc|}
\hline$M(H)$ & $\Gamma(H)$ & $B R(H \rightarrow Z Z)$ & $Z Z \rightarrow H$ & $W W \rightarrow H$ & $g g \rightarrow H$ \\
\hline 125 & 0.0056 & $1.89 \times 10^{-2}$ & 0.87 & 1.7 & 7.1 \\
140 & 0.0098 & $5.57 \times 10^{-2}$ & 2.4 & 4.7 & 19. \\
160 & 0.097 & $3.46 \times 10^{-2}$ & 1.2 & 2.6 & 10. \\
200 & 1.40 & 0.254 & 6.8 & 14. & 64. \\
400 & 30.0 & 0.262 & 2.6 & 5.2 & 51. \\
800 & 269.0 & 0.297 & 0.64 & 1.4 & 3.4 \\
\hline
\end{tabular}

Table 1: A summary of properties of the Standard Model Higgs as defined in PYTHIA. For each mass value, the width and branching ratio for $Z Z$ is given, along with the production cross section in $f b$, including the branching ratios for $Z$ decaying to $e$ or $\mu$ pairs. A top quark mass of $150 \mathrm{GeV}$ is assumed.

The properties of the Higgs which are embodied in PYTHIA are summarized in Table 1. The branching ratios agree well with the curves shown in Fig. 2.

The PAPAGENO generator was used to efficiently generate leptons arising from heavy fiavor decay's $(t \bar{t}, Z+b \bar{b}$, and $Z+t \bar{t}$ ). This generator contains a reasonably accurate model for the sequential decay of heavy quarks to leptons, but does not provide an accurate estimate for the flow of hadronic energy arising from the quark fragments or the underlying event and so cannot be used to estimate the effects of isolation.

The resolution, acceptance, and efficiency of the SDC detector used in this study are described in Ref. [10]. Pilenup events are included assuming an SSC luminosity of $10^{33}$ $\mathrm{cm}^{-2} \mathrm{sec}^{-1}$ (PYTHIA allows generation of additional minimum bias events for a given luminosity - only non-diffractive events have been used here). The generated particles were tracked through a simplified detector geometry, including the $2.0 \mathrm{~T}$ magnetic field, using GEANT. They were then allowed to interact in the calorineter using parametrizations for the transverse and longitudinal profiles of electromagnetic and hadronic showers. All plots have event rates corresponding to one standard SSC year of running (defined to be $10^{40} \mathrm{~cm}^{-2}$ or $10 \mathrm{fb}^{-2}$ ) ualess otherwise stated. 


\section{Background Rejection using Isolation}

In order to study the background rejections and signal efficiencies as a function of the lepton isolation requirement, we have used a sample of $t \bar{t}$ and $H \rightarrow 4 \ell$ events from PYTHIA. The Peterson fragmentation for heavy quarks was tuned to agree with recent ALEPH measurements [11]. These simulations include the effects of shower spreading and the magnetic field, and they assume the standard calorimeter segmentation of $\Delta \phi=$ $\Delta \eta=0.05$. We compute the amount of transverse energy in a cone of radius $R$ (in $\eta-\phi$ space) around the lepton direction and then remove the lepton energy itself. For muons, this is straightforward since one cell will almost always contain all of the lepton energy, whereas for electrons, many additional cells will bave some lepton energy ${ }^{2}$. For electrons, it is likely that test bearn information, combined with the actual impact point of the electron deduced from the tracking system, will be used to compute the energy deposition expected in cells near the impact point. When the expected energy deposition is below some threshold (determined by the amount of underlying event energy in the cell), then the predicted value rather than the observed value will give a more accurate determination of the actual lepton energy in that cell. The remaining energy is attributed to the underlying event and included in the isolation sum. Here, we use a somewhat idealized algorithm. We include in our isolation sum those energy depositions from the lepton that contribute less than $1 \mathrm{GeV}$ of $E_{t}$ in a single cell (GEANT tells us which energy in each cell is associated with which incident particles). This algorithm leaves of order 1 $\mathrm{GeV}$ of electron $E_{\mathrm{z}}$ behind in the isolation cone (this is probably a pessimistic estimate), thus creating a pedestal for the isolation measurement. We note that in a realistic SSC calorimeter, this pedestal may be overwhelmed by effects arising from the signal shaping in the calorimeter readout, which is neglected in the present simulation [12].

The isolation requirement has been studied for cone sizes varying from $R=0.1$ up to $R=0.7$, but the range which is most interesting for the current study is $R=0.2$ to $R=0.4$. The variable which has been chosen is the excess $E_{8}$ deposit in the cone (in $\mathrm{GeV}$ ), rather than a variable which is scaled by the lepton $p_{t}$. The argument is that the energy in the cone for signal events should be roughly constant (ignoring remnants of the lepton subtraction), whereas that for the background should increase with lepton $p_{t}$. Therefore, the fixed cut should have a constant efficiency and a monotonically improving rejection as a function of the lepton $p_{i}$, rather than the other way around. A series of figures are shown, and summarized in Table 2, to indicate how the isolation works for

\footnotetext{
${ }^{2}$ This is especially true at larger values of $\eta(\sim 2.5)$ where the electromagnetic shower size is not negligible on the scale of the cell size.
} 


\begin{tabular}{|cc|cccc|}
\hline$p_{t}$ Range & Radius & $Z$ Parent & $W$ Parent & $b$ Parent & $c, d$, or $u$ Parent \\
\hline \multirow{3}{*}{$10<p_{t}<20$} & 0.2 & $0.98 \pm 0.005$ & $0.94 \pm 0.02$ & $0.16 \pm 0.02$ & $0.013 \pm 0.005$ \\
& 0.3 & $0.96 \pm 0.008$ & $0.86 \pm 0.03$ & $0.07 \pm 0.01$ & $0.006 \pm 0.003$ \\
& 0.4 & $0.93 \pm 0.01$ & $0.78 \pm 0.03$ & $0.04 \pm 0.01$ & $0.004 \pm 0.003$ \\
\hline \multirow{3}{*}{$20<p_{t}<30$} & 0.2 & $0.99 \pm 0.003$ & $0.95 \pm 0.01$ & $0.05 \pm 0.01$ & $0.005 \pm 0.005$ \\
& 0.3 & $0.97 \pm 0.006$ & $0.88 \pm 0.02$ & $0.01 \pm 0.005$ & $<0.005$ \\
& 0.4 & $0.94 \pm 0.01$ & $0.81 \pm 0.02$ & $0.005 \pm 0.004$ & $<0.005$ \\
\hline & 0.2 & $0.98 \pm 0.005$ & $0.95 \pm 0.01$ & $0.02 \pm 0.01$ & $<0.008$ \\
$30<p_{t}<50$ & 0.3 & $0.95 \pm 0.007$ & $0.89 \pm 0.01$ & $<0.003$ & $<0.008$ \\
& 0.4 & $0.91 \pm 0.01$ & $0.83 \pm 0.02$ & $<0.003$ & $<0.008$ \\
\hline & 0.2 & $0.96 \pm 0.007$ & $0.95 \pm 0.007$ & $0.004 \pm 0.004$ & - \\
$50<p_{t}<150$ & 0.3 & $0.92 \pm 0.001$ & $0.89 \pm 0.01$ & $<0.004$ & - \\
& 0.4 & $0.8 \tilde{0} \pm 0.01$ & $0.80 \pm 0.01$ & $<0.004$ & - \\
\hline
\end{tabular}

Table 2: A summary of the observed efficiencies for detecting electrons with differen and the radius of the surrounding cone. Leptons are accepted if there is less than $5 \mathrm{GeV}$ of transverse energy in this cone. The " $Z$ parent" column refers to leptons arising from $H \rightarrow Z Z^{*}$ with $M_{H}=140 \mathrm{GeV}$. The other columns are for $t \bar{l}$ events with $M_{\text {top }}=150 \mathrm{GeV}$. Some entries are $68 \%$ confidence limits based on no observed events.

different bins in lepton $p_{t}$ and for different classes of leptons. The lepton class was defined based on the process and the identity of the lepton's parent.

The first distribution in each set of four is for leptons coming from a sample of 5000 $H \rightarrow 22$ * events, assuming $M_{H}=140 \mathrm{GeV}$. The other three distributions are for leptons coming from a sample of $50,000 t \bar{t}$ events, where the parent was a $W$, a $b$ quark, or a $c$ or lighter quark. Figure 3 shows distributions of the isolation variable for the lowest $p_{t}$ bin defined in this study $\left(10<p_{1}<20 \mathrm{GeV}\right)$ for a small cone of $R=0.2$. This figure indicates a problem that occurs when trying to reject low $p_{t} b$ quark backgrounds, namely the $b$ decay products are not tightly collimated, and a small cone size may often not include them, thereby leading to large numbers of "isolated" background leptons. Figure 4 shows the distributions for the same $p_{i}$ bin with a larger cone of $R=0.3$. Already, it is evident that the rejection is much improved (a factor of two). This emphasizes the need for relatively large isolation cones for low $p_{t}$ leptons, placing stringent constraiuts on the allowed calorimeter noise contributions (the current requirement of less than $1 \mathrm{GeV}$ in a 
cone of radius 0.15 at a luminosity of $10^{33} \mathrm{~cm}^{-2} \mathrm{sec}^{-1}$ is somewhat marginal for these low $p_{4}$ leptons).

In order to visualize the effect of the isolation cut, it is simpler to examine the integral distributions shown in Fig. 5, where the fraction of leptons of a given class passing a given isolation cut may be read off directly. A cut of $5 \mathrm{GeV}$ leads to the efficiencies given in Table 2. Raising this cut to $10 \mathrm{GeV}$ would decrease the background rejections by a factor of 2-3 for the assumed luminosity. The rejection of non-isolated leptons becomes easier as the lepton $p_{t}$ increases, due to the increased energy deposited in the isolation cone by the heavy quark decay products. This is apparent in Fig. 6, which contains leptons in the range $30<p_{t}<50 \mathrm{GeV}$. The distribution of $E_{t}$ in the cone is shifted further away from zero, increasing the rejection for a cut with fixed efficiency for the signal leptons.

In the remainder of this study, we will conservatively assume that the isolation cut reduces the rate of leptons from $b$ (and all lighter quarks) by a factor of 10 for each lepton. At design luminosity, assuming a cone size of $R=0.3$, one could achieve close to an additional factor of ten per lepton for most values of $p_{t}$. Extrapolating this performance to luminosities of $10^{34} \mathrm{~cm}^{-2} \mathrm{sec}^{-1}$ requires a more detajled consideration of the signal processing aspects of the problem; proper shaping of the readout changes the linear growth of pile-up noise with luminosity into a (roughly) square-root growth (for shaping times which are larger than the crossing time, one removes the "average" pile-up, leaving fluctuations about the average as the dominant effect). Further studies in this area will be carried out in the next few months as part of the physics assessment program. 

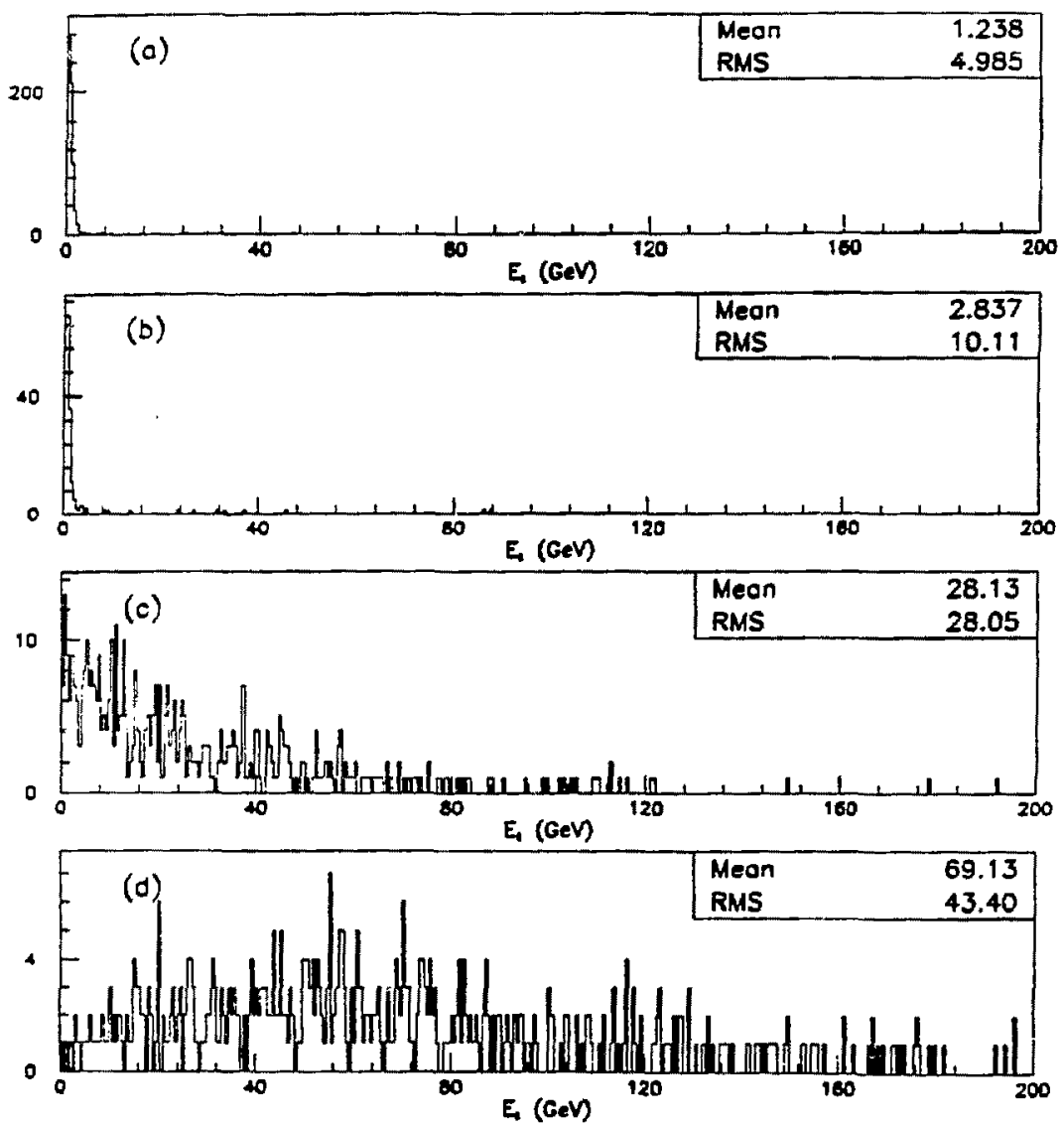

Figure 3: The distribution of $E_{8}$ in a cone of radius $R=0.2$ for different classes of electrons. The electrons are all in the range $10<p_{t}<20 \mathrm{GeV}$.

(a) Electrons from $H \rightarrow Z Z^{-}$for $M_{H}=140 \mathrm{GeV}$.

(b) $\mathrm{H}^{\prime}$ electrons coming from $t$ quark decays $\left(M_{\mathrm{lop}}=150 \mathrm{GeV}\right)$.

(c) b electrons coming from $t$ quark decays.

(d) $c$ (or $u, d$ ) electrons coming from $t$ quark decays. 

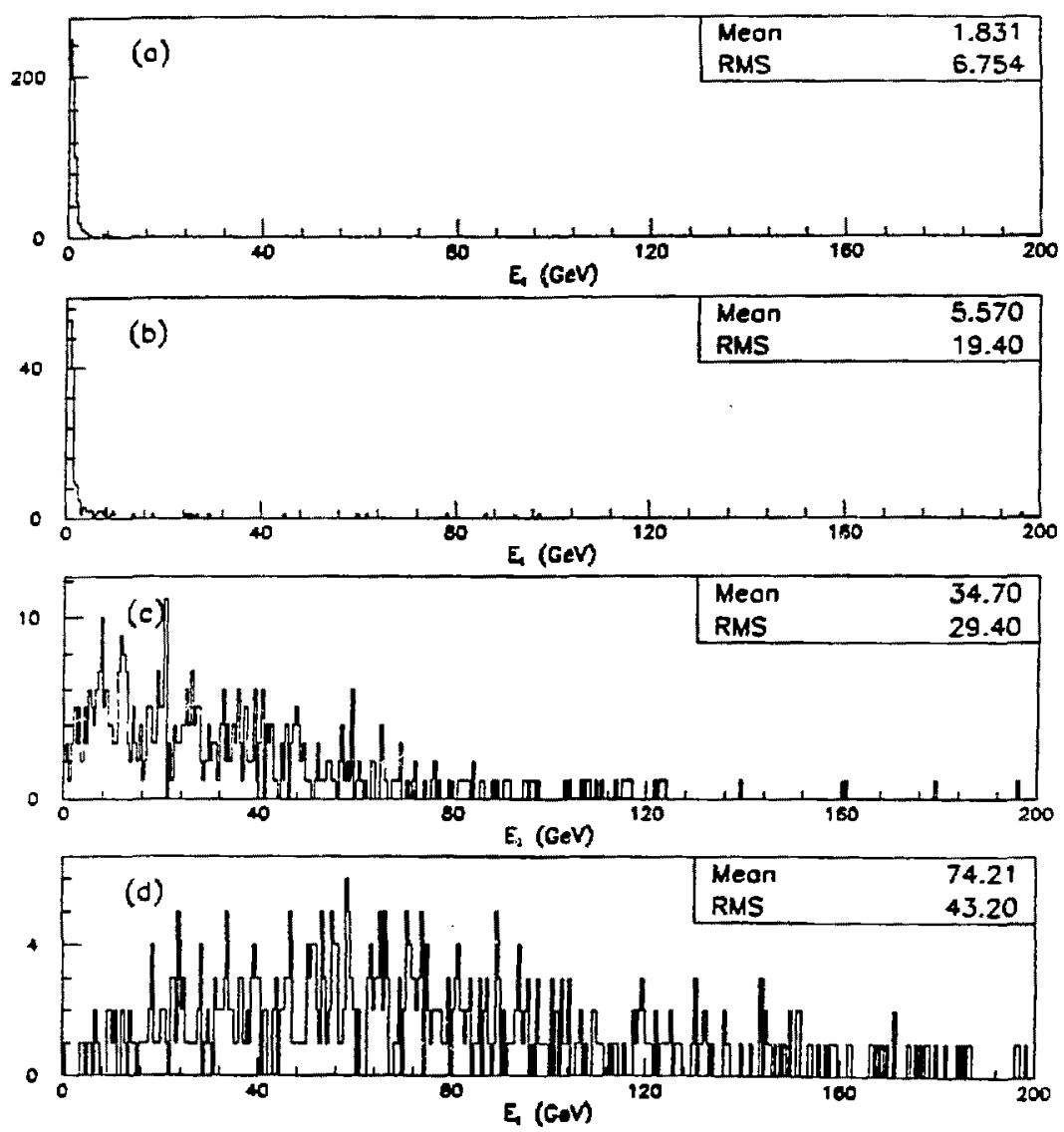

Figure 4: The distribution of $E_{\mathrm{z}}$ in a cone of radius $R=0.3$ for the same electron $p_{t}$ range displayed in Fig. 3 . The individual distributions also have the same significance as those in Fig. 3. 

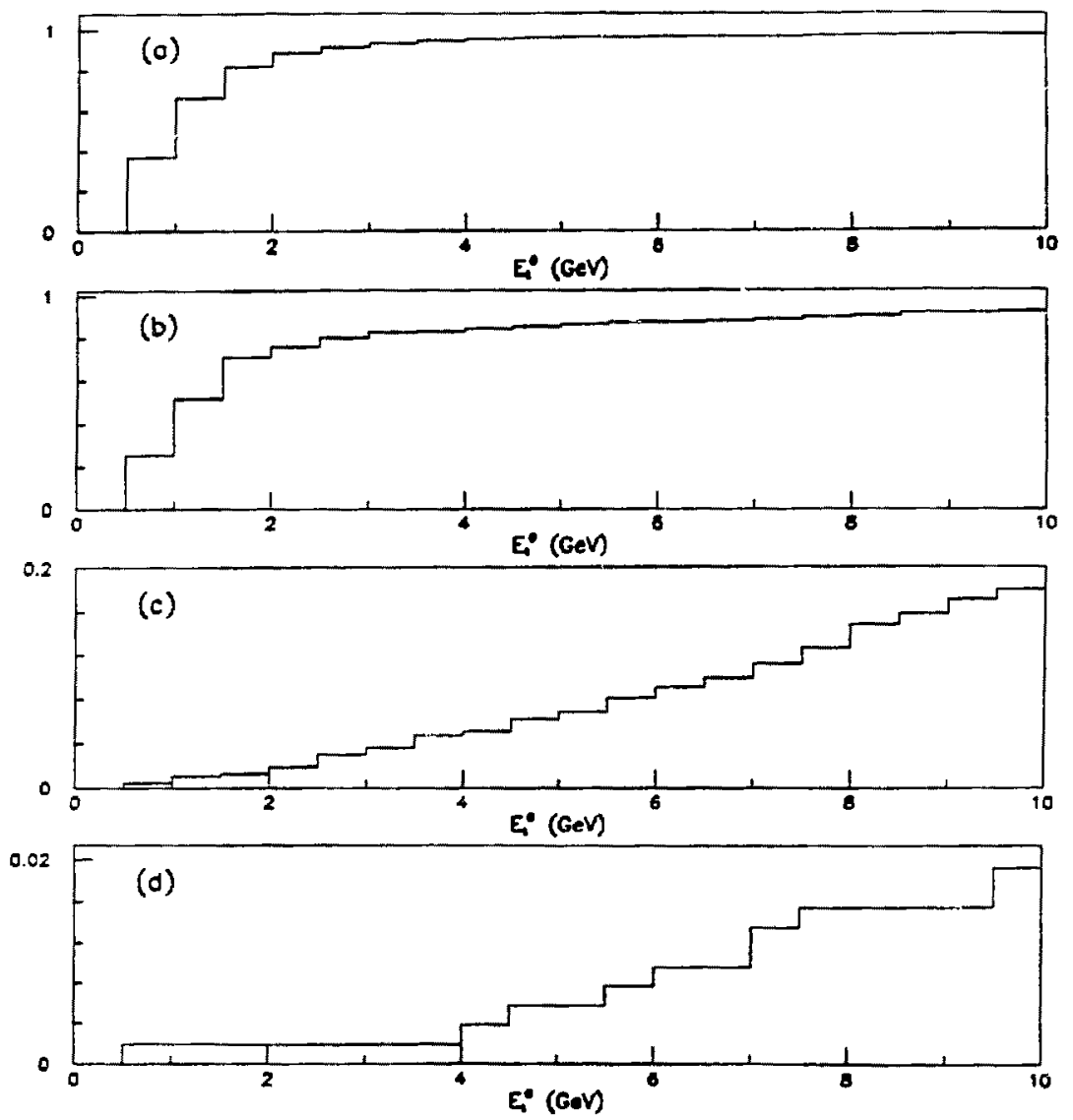

Figure 5: The integral aistribution of $E_{t}$ in a cone of radius $R=0.3$. The plot shows the fraction of events with $E_{t}>E_{t}^{0}$ as a function of $E_{\mathrm{l}}^{0}$. The electrons are all in the range $10<p_{1}<20 \mathrm{GeV}$ and the individual distributions bave the same sigirificance as those in Fig. 3. 

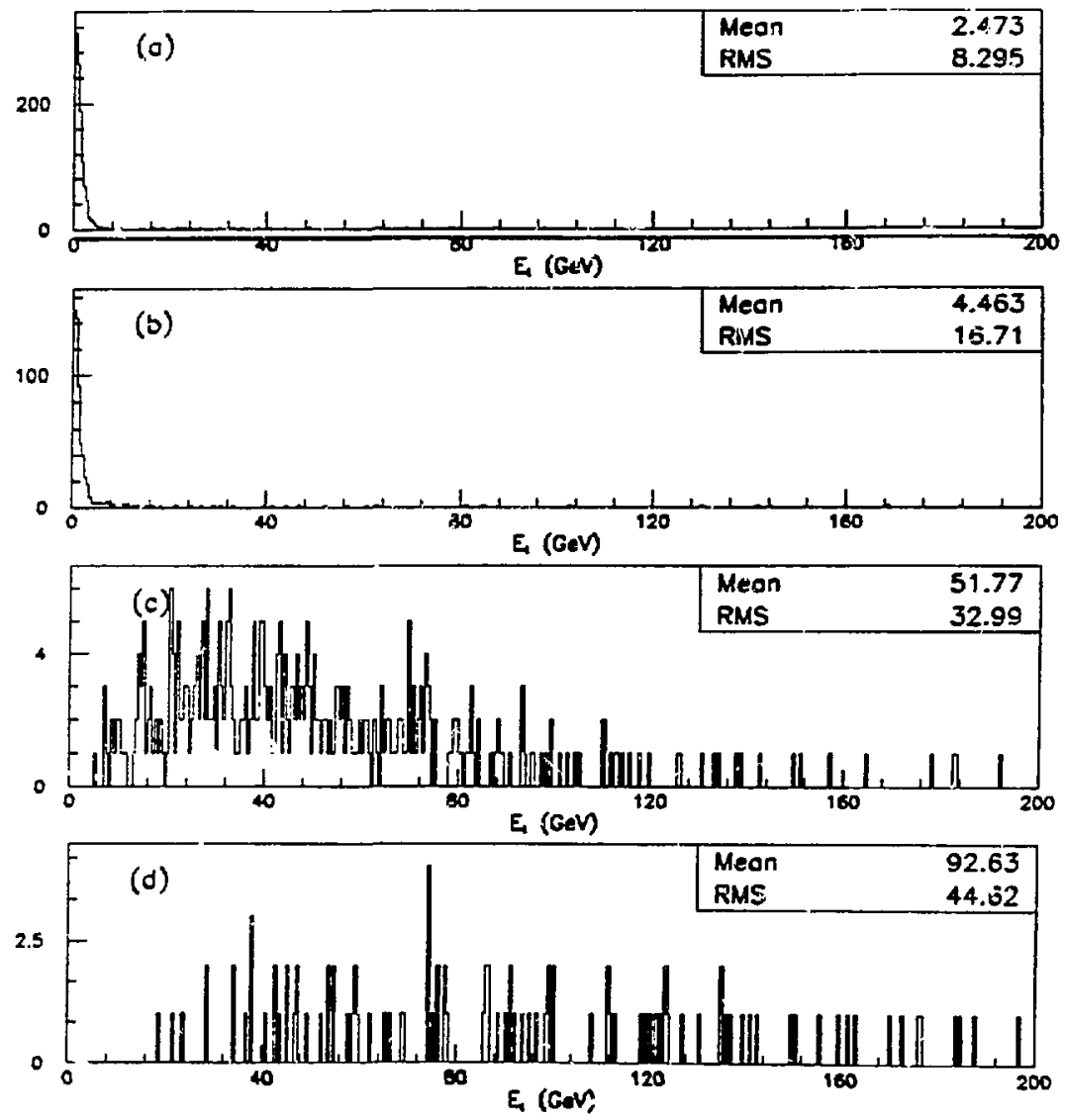

Figure 6: The distribution of $E_{t}$ in a cone of radius $\boldsymbol{R}=\mathbf{0 . 3}$ for electrons in the $p_{t}$ range $30<p_{t}<50 \mathrm{GeV}$. The individual distributions bave the same significance as those in Fig. 3. 


\section{Higgs with Mass below $2 M_{Z}$}

In this case, the transverse momentum of the $Z$ 's (real or virtual) is too small for the final state $\ell^{+} \ell^{-} \nu \nu$ to be useful. We therefore only consider the final state $\ell^{+} \ell^{-} \ell^{+} \ell^{-}$. For a Higgs mass of $140 \mathrm{GeV}$, there are approximately 250 events in final states with e's or $\mu$ 's in one standard SSC year.

There are four potential sources of lepton backgrounds (by requiring four identified leptons, all non-leptonic backgrounds become negligible). First, the production of $Z Z^{*}$ or $Z_{\gamma^{*}}$ (here * indicates a virtual particle) firal states from either $g \bar{q}$ or $g g$ initial states can give rise to the $4 \ell$ final state. The rate from the $q \bar{q}$ process is small [13]. The rate from $g g$ initial states is not known; we have increased the rate from $q \bar{q}$ by a factor of 1.65 to estimate its effect. ${ }^{3}$ This estimate is based upon comparisons of the calculated rates for $q \bar{q} \rightarrow Z Z$ and $g g \rightarrow Z Z$ at larger $Z Z$ invariant masses (see Ref. [17]). Next, there are backgrounds from final siates containing a $Z$ aud a pair of heavy quarks $(Z+b \bar{b}$ or $Z+t \bar{t}$ ), where semileptonic decays of the $t$ and $b$ quarks give rise to additional leptons. While the cross-section for the former process is much greater than she latter, the leptons from $t$ decay tend to be isolated while those from $b$ decay are not. Finally, there is the contribution from the $t \bar{t}$ final state. Four semileptonic decays resulting from this final state will give rise to a potential background. Two of the leptons (from the decays of $t$ and $\bar{t}$ ) are likely to be isolated, while the others are not. Hence the isolation requirement is very effective in removing this background.

The signal events have one pair of leptons whose invariant mass reconstructs to the $Z$ mass. Unlike the other backgrounds, the $t \bar{t}$ final state does not bave such a pair of leptons. Hence, it can be reduced by requiring that there be one pair of leptons whose invariant mass reconstructs to the $Z$ mass. The background rejection achieved by this requirement will depend on the resolution of the detector. Once the resolution is comparable to the natural width of the $\boldsymbol{Z}$ no further improvements in resoluticn will reduce this background. A further rejection can be obtained by removing like-sign lepton pairs which are produced by the heavy fiavor decays discussed above. By using the SDC nagnetic field for this purpose, one obtains a reductirn of roughly 1.5 for the $Z+b \bar{b}$ and $Z+t \bar{t}$ backgrounds, and roughly 2 for the $t \bar{t}$ background.

The rejection defined in the previous section has been applied to these backgrounds in the following (conservative) manner : $t \bar{t}$ and $Z+b \bar{b}$ cross sections are reduced by a factor $10^{2}$ (this assumes that only 2 of the 4 leptons come from $b$ or lighter quark decays), and

\footnotetext{
${ }^{3}$ The rate for the process $g g \rightarrow Z_{\gamma}$ (on-shell photon) is known [14]; it is approximately $20 \%$ of that for $q \bar{q} \rightarrow Z_{\gamma}$. Hence our background may be an overestimate.
} 
the $Z+t \bar{t}$ cross section is left unchanged (this assumes that all 4 leptons come from $Z$ 's or $W$ 's). The resulting background's, including all of the kinematic cuts defined below, for the mass region $100<M_{z Z^{*}}<180 \mathrm{GeV}$, are shown in Fig. 7 .

Events are triggered by requiring two leptons with transverse momentum greater than $20 \mathrm{GeV}$. This results in a trigger effieiency of $\sim 50 \%$ (70\%) for a Higgs mass of $120(160)$ $\mathrm{GeV}$. Figure 8 show the acceptance of the detector as a function of the minimum lepton transverse momentum for several values of the rapidity coverage (the default coverage is $|\eta|<2.5)$. In this study we assume that leptrins of $p_{t}$ less than $10 \mathrm{GeV}$ cannot be detected with adequate background rejection. Hence it can be seen that requiring that the four leptons be in the SDC fiducial volume and pass the triggering (2 leptons with $p_{t}>20$ $\mathrm{GeV}$ ) and detection (4 leptons with $p_{t}>10 \mathrm{GeV}$ ) requirements results in an efficiency of $\sim 25 \%(45 \%)$ for a Higgs mass of $120(160) \mathrm{GeV}$. To simplify the analysis, only the four highest $p_{t}$ leptons inside the acceptance are used in the subsequent calculations (this also provides some further rejection against backgrounds which produce more than four leptons). The charge-zero lepton pair with mass closest to $M_{Z}$ was required to satisfy $M_{\ell}=M_{Z} \pm 10 \mathrm{GeV}$. The other lepton pair was required to have $M_{\ell}>20 \mathrm{GeV}$ to remove the $q \bar{q} \rightarrow Z \gamma^{*}$ background. All kinematic quantities were calculated from the tracking information, which provides slightly bettes electron resolution than the calorimeter for the momenta relevant for this process $\left(\sigma\left(M_{Z Z}\right)=1.0 \mathrm{GeV}\right.$ instead of $1.3 \mathrm{GeV}$ for $M_{H}=140$ $\mathrm{GeV}$ ). However, note that these electrons, will suffer large bremsstrahlung losses in the SDC magnetic field (the CDF $Z \rightarrow e^{+} e^{-}$peak is silifted by about $2 \mathrm{GeV}$ due to this effect), making accurate reconstruction more difficult. The final determination of the mass will need to use both the tracking and calorimeter measurements.

In order to reject background for final states involving $t$ and $b$ quarks, we require isolated leptons. Applying the isolation requirement discussed above to all 4 !eptons in the signal results in an additional loss of roughly $4 \%$ of the events for each isolated lepton (a total efficiency of $85 \%$ for all four leptons). Finally there is a loss due to the efficiency with which the detector can reconstruct electrons and muons. This is assumed to be $85 \%$ for each lepton unless the leptons reconstruct to a $Z$, in which case we assume an efficiency of $95 \%$ for the second of the two leptons. The total effect of acceptance and efficiency is to reduce the aumber of events from 250 (produced) to roughly 40 (observed) for a Higgs of mass $140 \mathrm{GeV}$.

Figure 9a sbows the reconstruction of a Higgs boson of mass 125,140 and $160 \mathrm{GeV}$, together with the expected backgrounds, using tracking measurements for the lepton momenta. The resolution of the SDC in the electron and muon channels is comparable for the range of transverse momenta relevant in this case and hence the signals in the different 

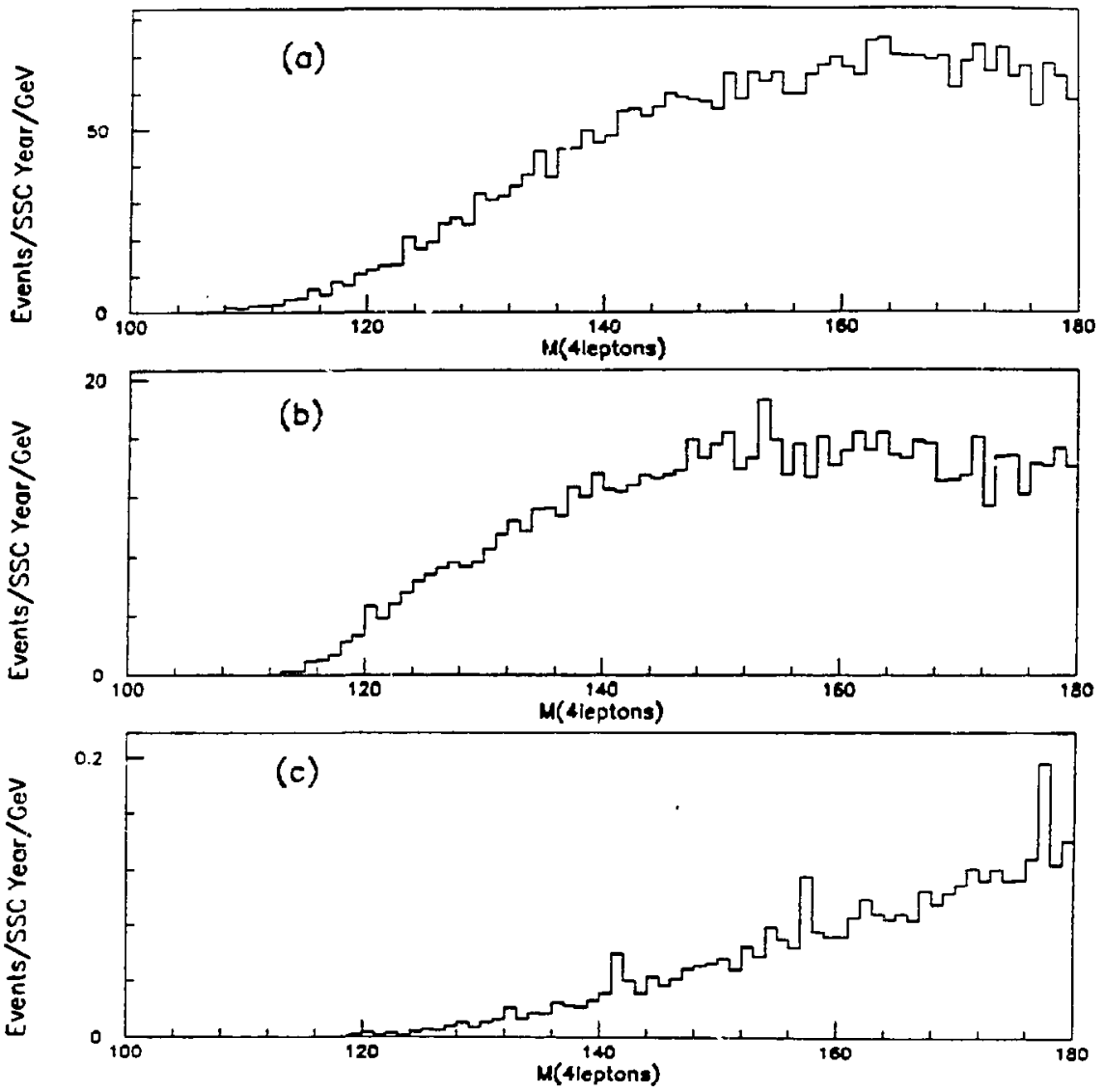

Figure 7: The invariant mass distribution $d N / d M_{4}$ for background events satisfying the kinematic cuts used in the $H \rightarrow Z Z^{*}$ analysis as a function of the four lepton invariant mass $M_{\mathbf{4} \ell}$ (no isolation cut has been applied). The events were generated by PAPAGENO using the standard resolution parametrizations.

(a) The distribution for $t \bar{t}$ events.

(b) The distribution for $Z+b \bar{b}$ events.

(c) The distribution for $Z+i \bar{t}$ events. 

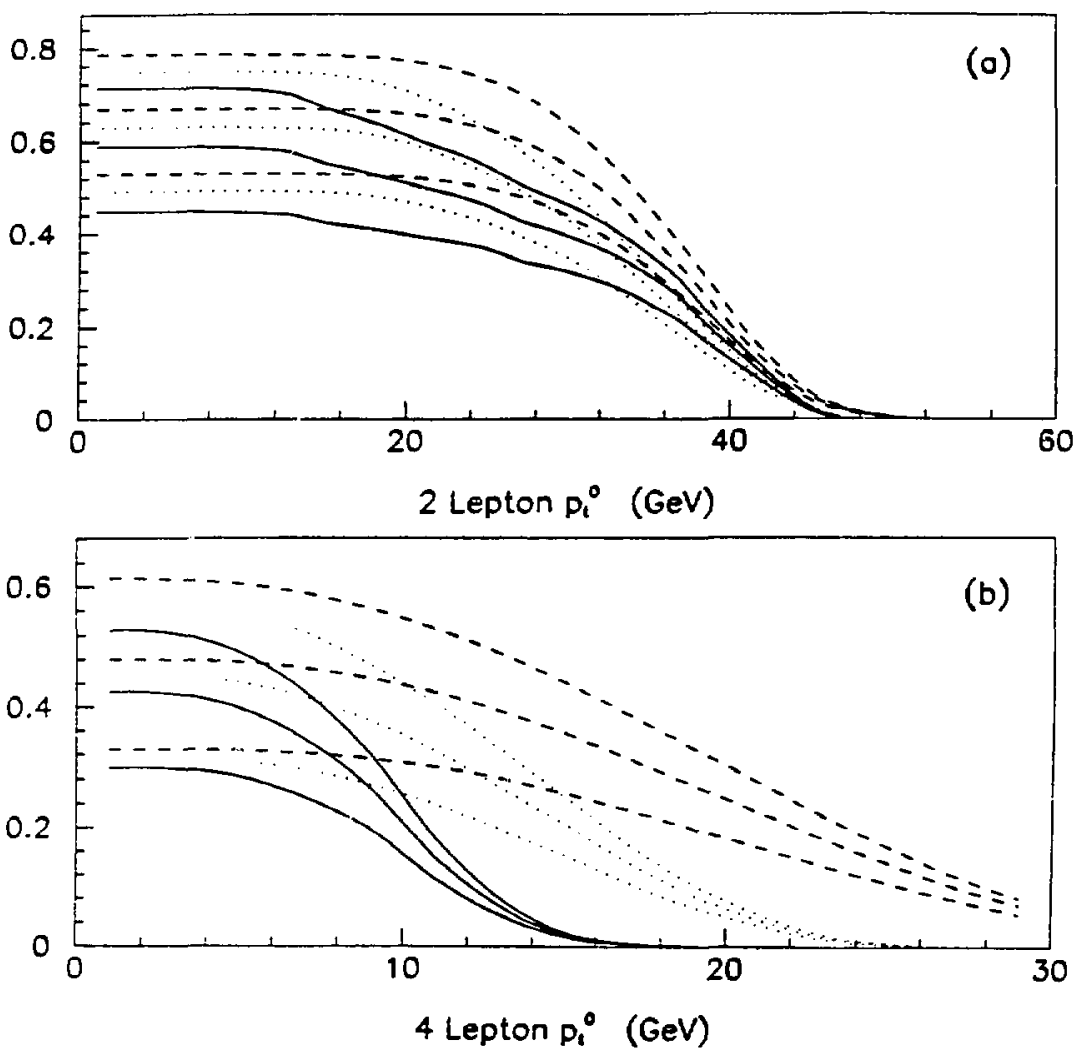

Figure 8: Families of acceptance curves for $H \rightarrow Z Z^{*}$, for $M_{H}=120 \mathrm{GeV}$ (solid), $140 \mathrm{GeV}$ (dotted), and $160 \mathrm{GeV}$ (dashed).

(a) The fraction of events with at least two leptons with $p_{t}>p_{0}$ as a function of po. Both leptons have $|\eta|<2.0$ (lower curve), 2.5 (middle curve), or 3.0 (upper curve).

(b) The fraction of events containing two leptons with $p_{1}>20 \mathrm{GeV}$ and $|\eta|<$ 2.5 plus two others with $p_{t}>p_{0}$ and $|m|<2.0$ (lower curve), 2.5 (middle curve), or 3.0 (upper curve). 
channels have been combined. Figure $9 \mathrm{~b}$ shows the same plot using calorimeter measurements for the electrons, thereby simplifying the reconstruction with some consequent loss in resolution.

The statistical significance of the peaks shown in Fig. 9a bas besn evaluated by counting the number of events predicted above the expected backgrounds in the vicinity of each peak (a region of $\pm 3 \mathrm{GeV}$ was used). The $M_{H}=140 \mathrm{GeV}$ and $M_{H}=160 \mathrm{GeV}$ peaks are unambiguous. There are 38 events with 3.5 expected background, and 30 events with 5 expected background, respectively, for one year of SSC running at nominal luminosity. The $M_{H}=125 \mathrm{GeV}$ peak has 8 events with 2 expected background, and consequently requires about 2 years of SSC running to establish a convincing signal. At lower masses the rates are very small due to the rapidly falling branching ratio into the $Z Z^{*}$ final state (see Fig. 2). Our requirement that leptons have $p_{t}>10 \mathrm{GeV}$ results in an acceptance that falls as the Higgs mass is reduced. An increase in the integrated luminosity in excess of $10^{40} \mathrm{~cm}^{-2}$ will enable the mass range to be extended slightly, but in order to probe Higgs masses much below $120 \mathrm{GeV}$ another method is needed [15]. 

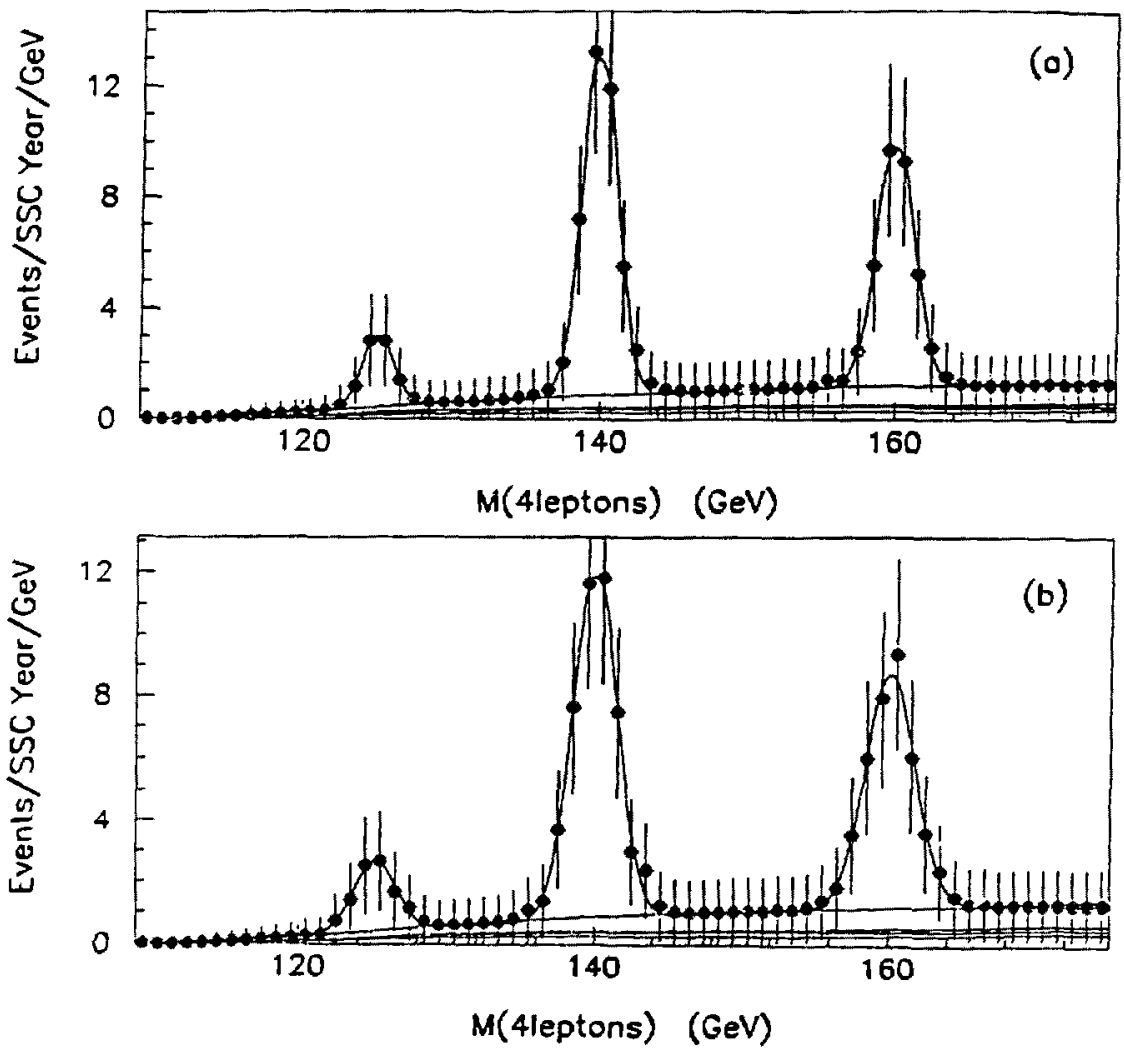

Figure 9: The reconstructed Higgs mass for $Z Z^{-}$decaying to $4 e, 4 \mu$, and $2 e 2 \mu$ with $M_{H}=125,140,160 \mathrm{GeV}$, including the expected backgrounds. The backgrounds curves are cumulative, and are (from lowest to highest): $q \bar{q} \rightarrow$ $Z Z^{*}$, multiplied by 1.65 to account for $g g \rightarrow Z Z^{*}, Z+b \bar{b}, Z+t \bar{t}$, and $t \bar{t}$.

(a) The invariant mass reccnstructed from tracking measurements.

(b) The invariant mass using calorimeter measurements for the electrons. 


\section{Higgs with Mass above $2 M_{Z}-$ the $\ell^{+} \ell^{-} \ell^{+} \ell^{-}$mode.}

In this section, we consider the specific cases of $M_{H}=200 \mathrm{GeV}, 400 \mathrm{GeV}$, and $800 \mathrm{GeV}$. Once we are above the $Z Z$ threshold, the signal consisis of two pairs of leptons each of which has an invariant mass consistent with the $Z$ mass (defined as $M_{Z} \pm 10 \mathrm{GeV}$ ). Again, we trigger on two isolated leptons of $p_{t}>20 \mathrm{GeV}$ and require that all four leptons bave $p_{t}>10 \mathrm{GeV}$. The background arises from the processes $q \bar{q} \rightarrow Z Z[16]$ and $g g \rightarrow Z Z[17]$. Backgrounds from the final states $Z+t \bar{t}, Z+b \bar{b}$, or $\bar{t}$ have also been considered. They are shown in Figs. 10-12, including all kinematic cuts, for the mass regions of interest. It is apparent that these backgrounds are small once we require that there are two lepton pairs whose invariant mass reconstructs to $M_{Z} \pm 10 \mathrm{GeV}$ (the backgrounds are much lower than those in Fig. 7 because we can require two lepton pairs close to the $Z$ mass). They become negligible once we make the additional requirement that the four leptons are isolated.

Figure 13 shows the reconstructed $Z$ mass for lepton pairs produced in the decay of an $800 \mathrm{GeV}$ Higgs. The charge-zero pair with invariant mass closest to the $Z$ mass is selected as the first $Z$ candidate, and the other lepton pair is taken to be the second $Z$ candidate. Figure 13 contains both pair masses, plotted separately for the individual lepton types. It indicates that the SDC resolution, as assumed in the LoI, is a good match to the 10 $\mathrm{Gel} Z$ mass window used in this analysis. In more quantitative terms, the acceptance for the $1 I_{Z} \pm 10 \mathrm{GeV}$ requirement on both pairs for the $4 \mu$ channel is reduced by $6 \%$ relative to that for the $4 e$ channel due to the standard muon resolution. The acceptance of the detector as a function of the minimum lepton transverse momentum for several values of rapidity coverage is shown in Fig. 14. It is evident that the $p_{t}$ threshold has little influence on the acceptance, whereas increasing the $\eta$ coverage from 2.5 to 3.0 would increase the acceptance by $\sim 20 \%$.

Figures 15-17 show the result of adding the signal and expected background for the three Higgs masses considered here. The electrons have been reconstructed from the calorimeter information (giving better resolutions in this kinematic regime), whereas the muons use the combined tracking and muon system resolutions. The different resolutions in these channels do not manifest themselves in significantly different $Z Z$ mass spectra, due to the large intrinsic width of the Higgs boson at these masses. The background from $q \bar{q} \rightarrow Z Z$ has been multiplied by 1.65 to take account of the $g g \rightarrow 2 Z$ process which is not included in the Monte-Carlos. In the case of Iliggs masses of 200 and $400 \mathrm{GeV}$, a clear peak above background can be seen and the rates are sufficient for a discovery to be made. In the case of a Higgs mass of $800 \mathrm{GeV}$, there are fewer signal events and no 

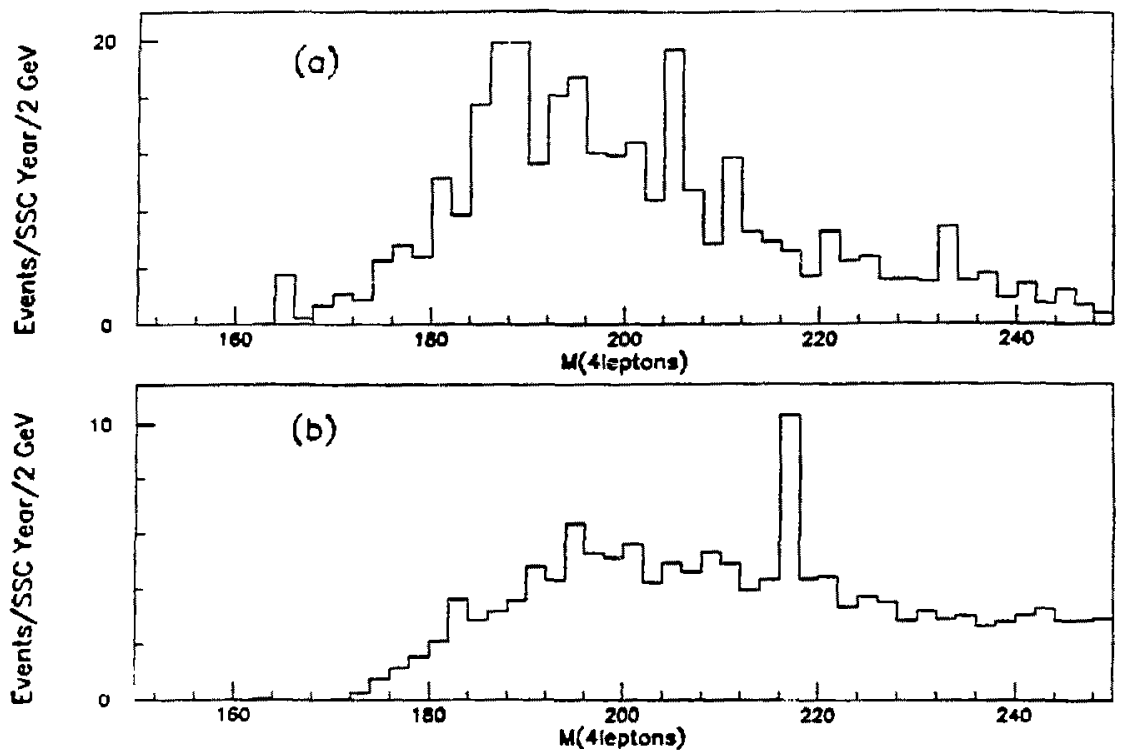

U.

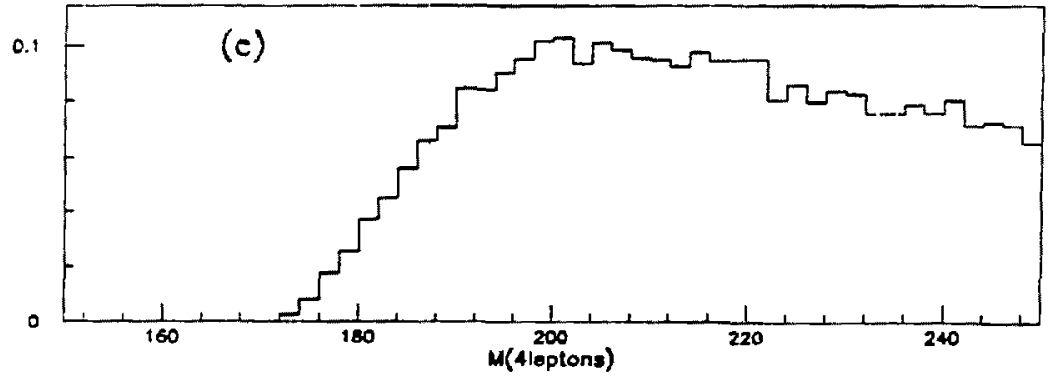

Figure 10: The invariant mass distribution $d N / d M_{4}$ for background events satisfying the kinematic cuts used in the $H \rightarrow Z Z$ analysis as a function of the four lepiou invariant mass $M_{1}$ for a Higgs mass of $200 \mathrm{GeV}$ (no isolation cut has been applied).

(a) The distribution for $t \bar{t}$ eveats where two pairs of oppositely charged leptons are required to have an invariant mass of $M_{Z} \pm 10 \mathrm{GeV}$.

(b) The distribution for $Z+b \bar{b}$ events.

(c) The distribution for $Z+t \bar{t}$ events. 

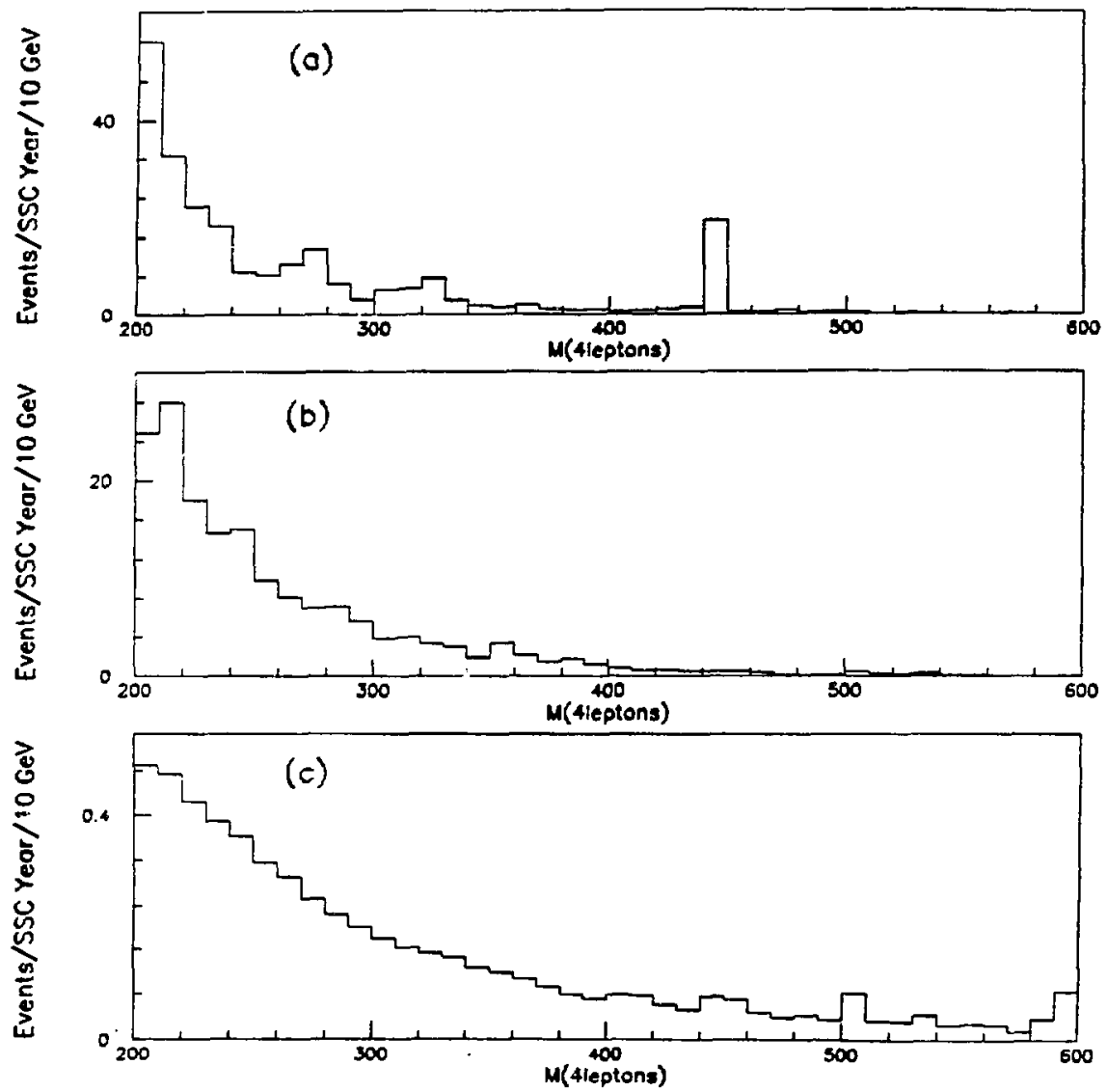

Figure 11: Same as Fig. 10 except for a Higgs mass of $400 \mathrm{GeV}$ (no isolation cut has been applied). 

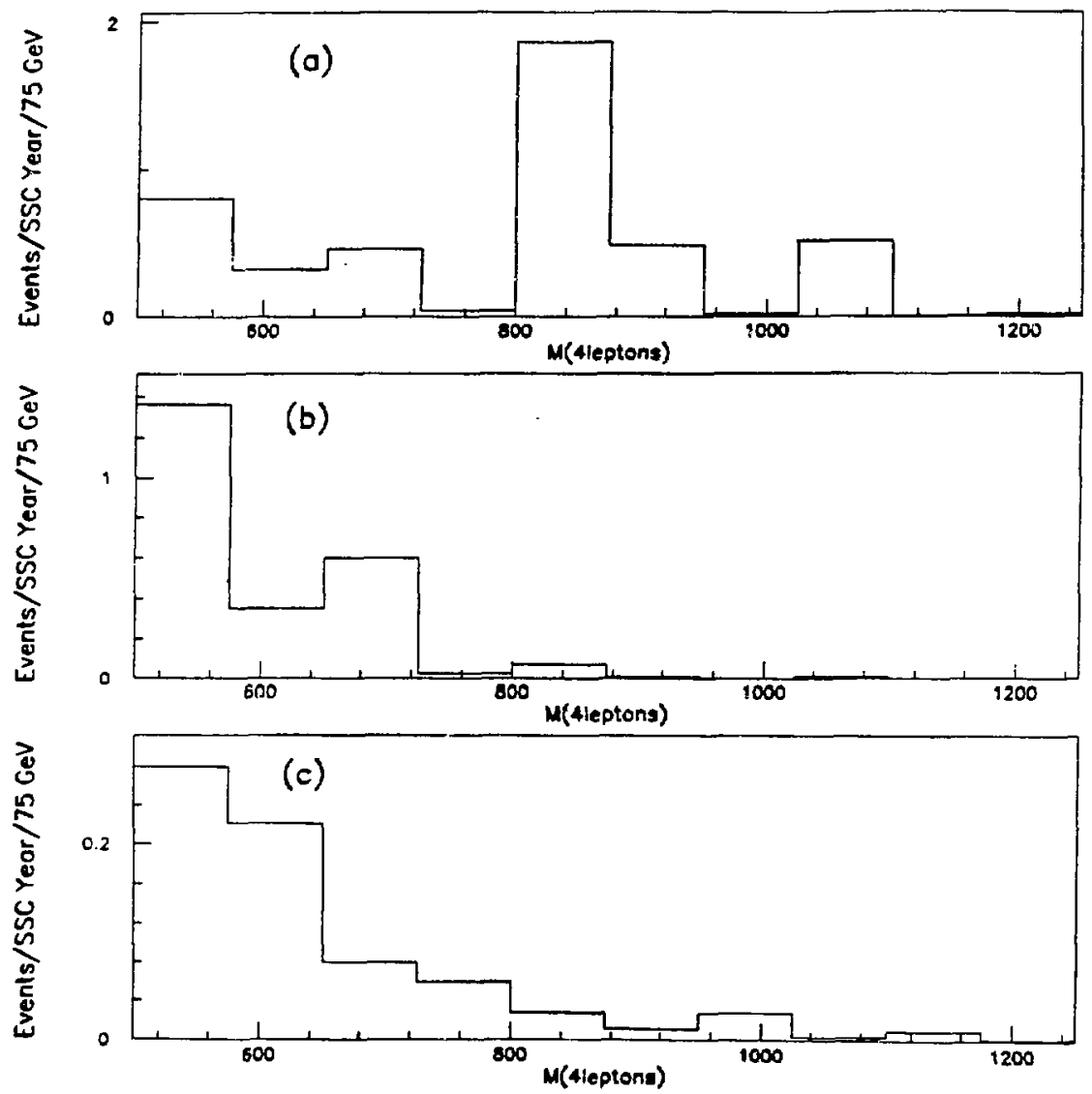

Figure 12: Same as Fig. 10 except for a Higgs mass of $800 \mathrm{GeV}$ (no isolation cut has been applied). 


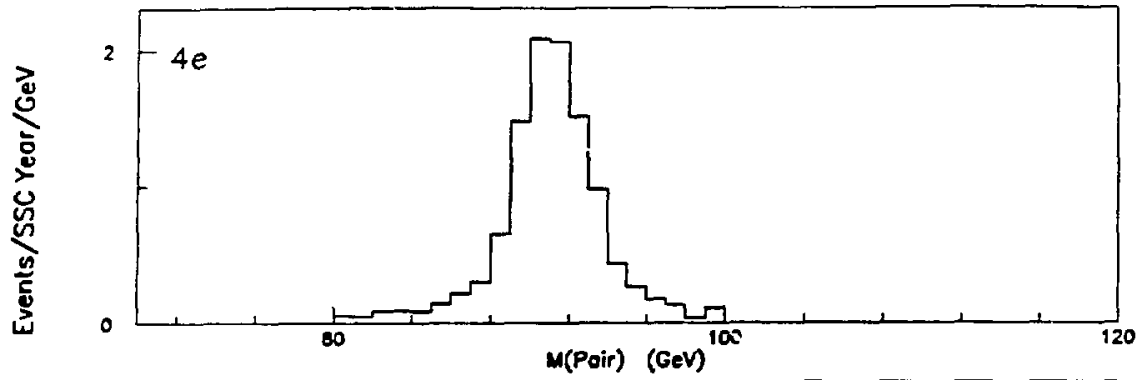

守
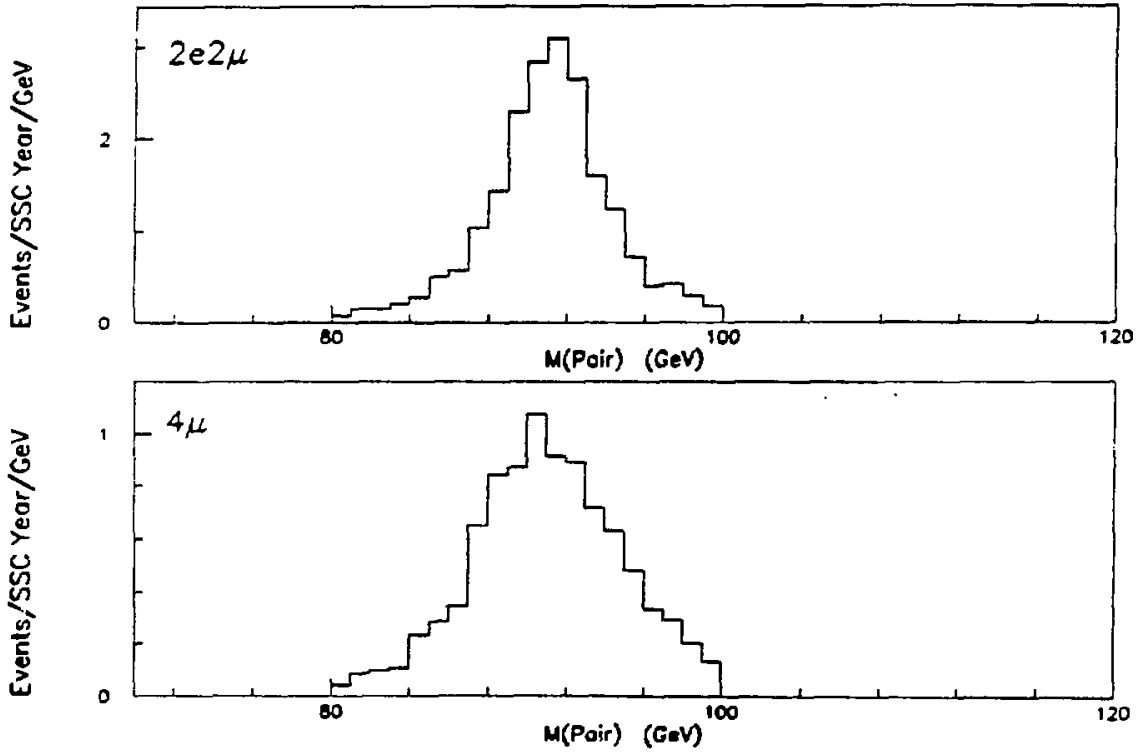

Figure 13: The invariant mass distribution for lepton pairs from $H \rightarrow Z Z$ events for a Higgs mass of $800 \mathrm{GeV}$, showing the $4 e, 2 e 2 \mu$, and $4 \mu$ channels separately. The tracking measurements are used for the rnuons, whereas the calorimeter measurements are used for the electrons. 

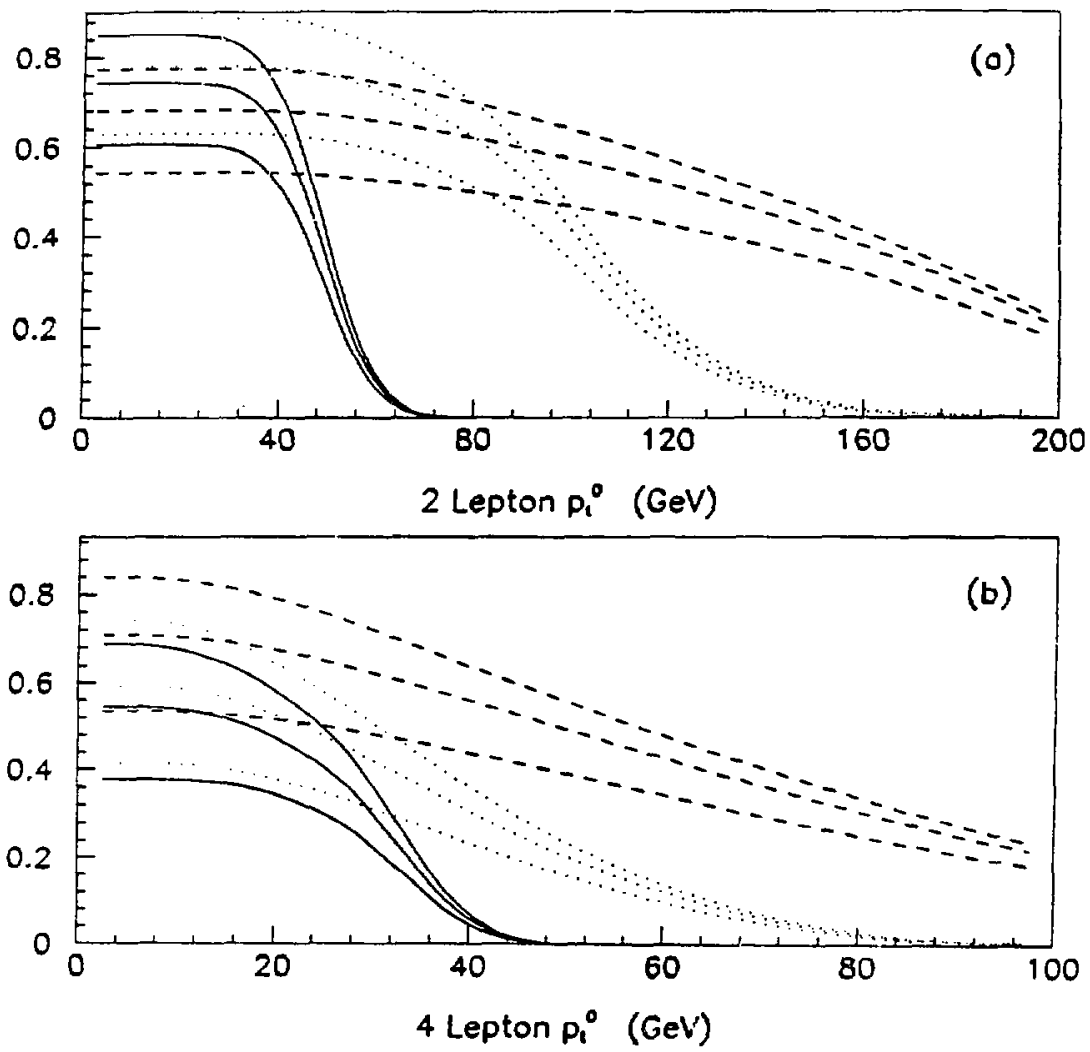

Figure 14: Families of acceptance curves for $H \rightarrow Z Z$, for $M_{H}=200 \mathrm{GeV}$ (solid), $400 \mathrm{GeV}$ (dotted), and $800 \mathrm{GeV}$ (dashed).

(a) The fraction of events with at least two leptons with $p_{t}>p_{0}$ as a function of $p_{0}$. Both leptons have $|\eta|<2.0$ (lower curve), 2.5 (middle curve), or 3.0 (upper curve).

(b) The fraction of events containing two leptons with $p_{t}>20 \mathrm{GeV}$ and $|\eta|<$ 2.5 plus two others with $p_{t}>p_{0}$ and $|\eta|<2.0$ (lower curve), 2.5 (middle curve), or 3.0 (upper curve). 
clear peak. We note that the line shape for an $800 \mathrm{GeV}$ Higgs is sufficiently broad (the intrinsic width is $270 \mathrm{GeV}$ ) that the mass distribution no longer peaks at $800 \mathrm{GeV}$, due to the falling parton flux. The peak of a relativistic Breit-Wigner with an energy dependent width will be shifted downwards by about $50 \mathrm{GeV}$ by the exponentially falling parton luminosity.

The signal to background ratio for the $800 \mathrm{GeV}$ Higgs can be improved by requiring that the transverse momentum of both of the $Z$ 's is more than $200 \mathrm{GeV}$. As is evident in Fig. 18, the background has been reduced with little loss in signal. The peak region contains 20 events with 6 expected background. To claim a signal in the $800 \mathrm{GeV}$ case, one must have confidence in the $Z Z$ rate at large $Z Z$ invariant masses that would be expected in the absence of a Higgs boson. The measured $Z Z$ rate at lower invariant masses can be used to reduce the uncertainties in the theoretical predictions for the $Z Z$. rate at large values of the invariant mass. The major uncertainties is this extrapolation arise from the structure functions and higher order QCD corrections [18]. We estimate that we can determine the background with an uncertainty of $20 \%$, and therefore the $M_{H}=800 \mathrm{GeV}$ signal would require 2-3 years of SSC running at the nominal luminosity to be sufficient for discovery. 


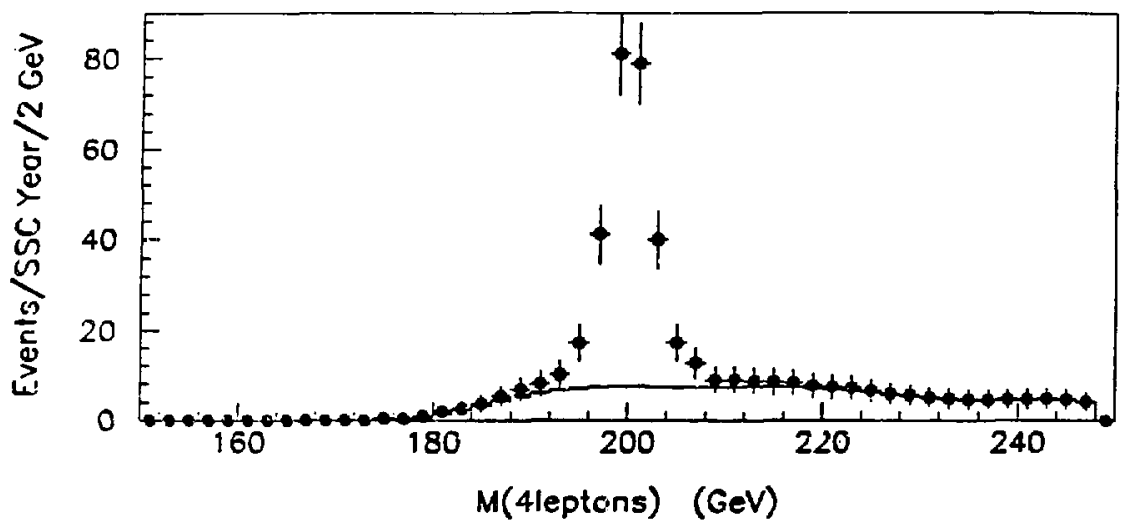

Figure 15: The $Z Z$ invariant mass distribution showing a peak due to a Higgs of mass $200 \mathrm{GeV}$. The two lepton pairs were both required to have $M_{\mu}=$ $M_{Z} \pm 10 \mathrm{GeV}^{r}$. The background curves have the same significance as those of Fig. 9, but the $Z Z$ background gives the only visible contribution.

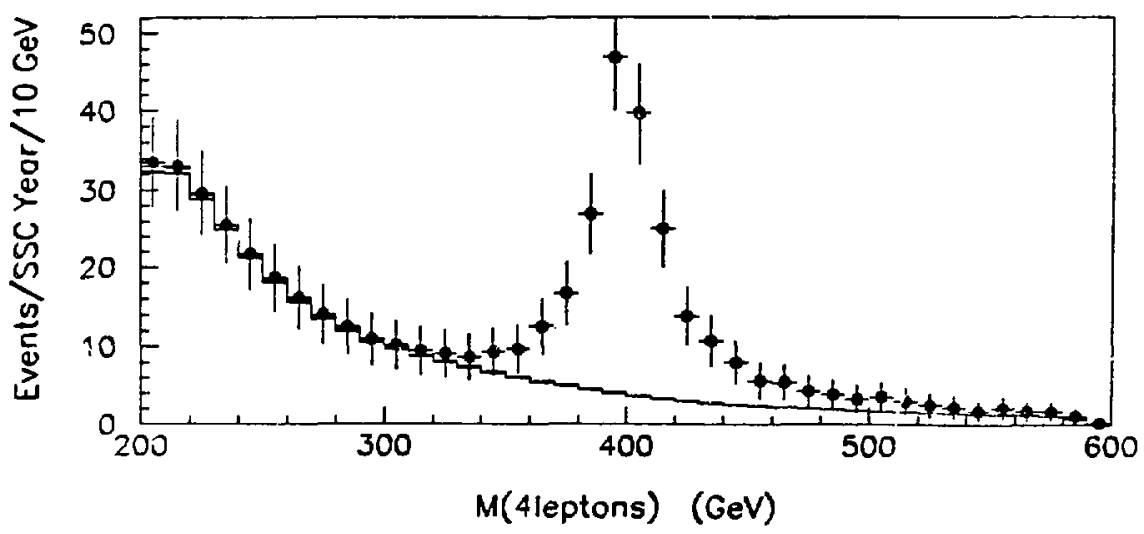

Figure 16: Same as Fig. 15, except that the Higgs mass is $400 \mathrm{GeV}$. 


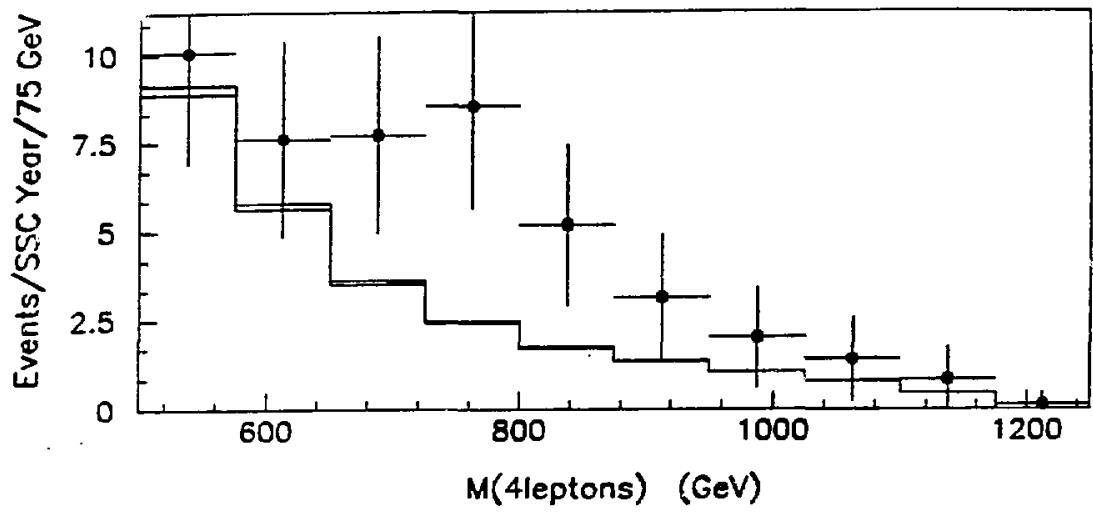

Figure 17: Same as Fig. 15, except that the Higgs mass is $800 \mathrm{GeV}$.

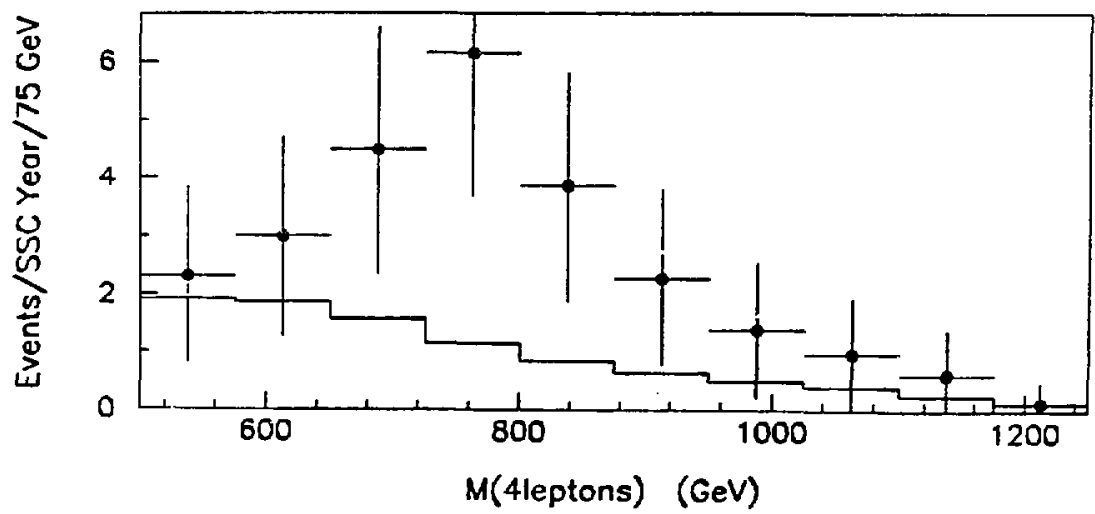

Figure 18: Same as Fig. 17, except that both $Z$ 's were required to satisfy $p_{\mathrm{r}}(Z)>200 \mathrm{GeV}$. 


\section{Higgs with mass above $2 M_{Z}$ - the $\ell^{+} \ell^{-} \nu \nu$ mode.}

In view of the limited rate at $M_{H}=800 \mathrm{GeV}$, we have looked at the channel with $e^{+} e^{-}$or $\mu^{+} \mu^{-}$and missing $E_{T}$ in order to extract the sigal from the decay $I \rightarrow Z Z \rightarrow \ell^{+} \ell^{-} \nu \bar{V}$. Here again one background is from $Z Z$ final states, but backgrounds arise from the $Z+j e t s$ final state where the jets are mismeasured or lost dowa the team hole, from $Z+$ heavy quarks where the missing $E_{t}$ arises from semileptonic decays and from $t \bar{t}$ events where two isolated leptons are produced that have an invariant mass close to the $Z$ mass. The missing $E_{T}$ resolution of our detector now plays a vital role in our ability to extract a signal. Figure 19 shows the contribution of the various backgrounds which we now discuss in detail.

The background from the $t \bar{t}$ final state is computed by requiring that there be a pair of isolated leptons (these arise almost exclusively from the decay $t \rightarrow b \ell v$ ) of opposite charge whose invariant mass is $M_{Z} \pm 20 \mathrm{GeV}$. The resolution of the SDC is somewhat better than this but the limited Monte-Carlo statistics prevent us from making a tighter cut. In this case the missing $E_{z}$ is mainly due to neutrinos and not to resolution effects.

In the case of the $Z+j$ ets background there are two detector effects that contribute to the background. The jet resolutions assumed for our detector [10] have very long nonGaussian tajls (modeled on those observed in the CDF detector [19]) and hence there is a non-negligible possibility that the jet will have its energy mismeasured by a very large amount and will give rise to a large apparent missing $E_{T}$. These tails are due $10 \mathrm{cracks}$ in the CDF detector as well as to neutrinos arising from semileptonic decays (mainly bottom and charm quarks). In order to reduce the background from the final state $Z+j e t s$ we have required that there be no jets in the event which are measured to have transverse energy above $300 \mathrm{GeV}$. This has a negligible effect on the signal but reduces this background by at least a factor of five at a missing $E_{T}$ of $300 \mathrm{GeV}$ or greater. If we remove the non-Gaussian tails from our jet resolutions or make them less severe [20], we can drop this jet veto since the background from $Z+j e t s$ is then no longer important. The second detector effect is that caused by jets in the forward region being lost out of the end of the forward calorimeter. In this context, it is worth remarking that a jet with transverse momentum of $300 \mathrm{GeV}$ at $\eta=4$ has an energy of $8.2 \mathrm{TeV}$ and hence a very small production rate.

There are several possible final states involving a $Z$ and heavy quarks. We have looked at the background from the $Z+t \bar{t}$ and $Z+b \bar{b}$ final states. 'There are two ways in which

\footnotetext{
'The process $g t \rightarrow \imath Z$, which is included in some Monte-Carlos is a very poor approximation to $g g \rightarrow 2 i \vec{t}$
} 
these processes can contribute. First, events can have $Z \rightarrow \nu \bar{\nu}$ decays and two leptons that have an invariant mass close to the $Z$ (we require $M_{Z} \pm 20 \mathrm{GeV}$ ) arising from the semileptonic heavy quark decays. Second, events can have $Z \rightarrow \ell \ell$ and missing $E_{T}$ arising from the heavy quark decays. Summing these contributions produces less than 3 events (without the jet veto) in the bin of Figure 20 with missing $E_{t}$ of $300 \mathrm{GeV}$ where there are about 50 signal events. The contributions from $Z \rightarrow \ell \ell$ are the dominant ones, with the top quarks giving abcut 2 events and the bottom quarks giving one event. By including the jet veto, the top contribution does not change much but the bottom contribution falls by at least a factor of ten (this estimate is limited by Monte-Carlo statistics).

Figure 20 shows the missing $E_{\mathrm{l}}$ distribution for events where we have required that there be an $e^{+} e^{-}$or $\mu^{+} \mu^{-}$pair with mass $M_{Z} \pm 20 \mathrm{GeV}$ and $p_{t}>250 \mathrm{GeV}$, and that there be no detected jets with $p_{t}>300 \mathrm{GeV}$. The two dominant detector dependent backgrounds are shown separately. It can be seen that these are negligible compared with the irreducible background from the $Z Z$ final state. Again, this method of searching for the Higgs is therefore limited by the production rate and not the background. The strateg: for extracting a signal from this final state is similar to that in the 4-charged lepton channel. Measurements of that channel and of the missing $E_{T}$ spectrum at lower values are used to reduce the uncertainties in the predicted missing $E_{T}$ spectrum at larger missing $E_{T}$ in the absence of a Higgs boson. The observed excess of events would convincingly establish the existence of the Higgs boson. 


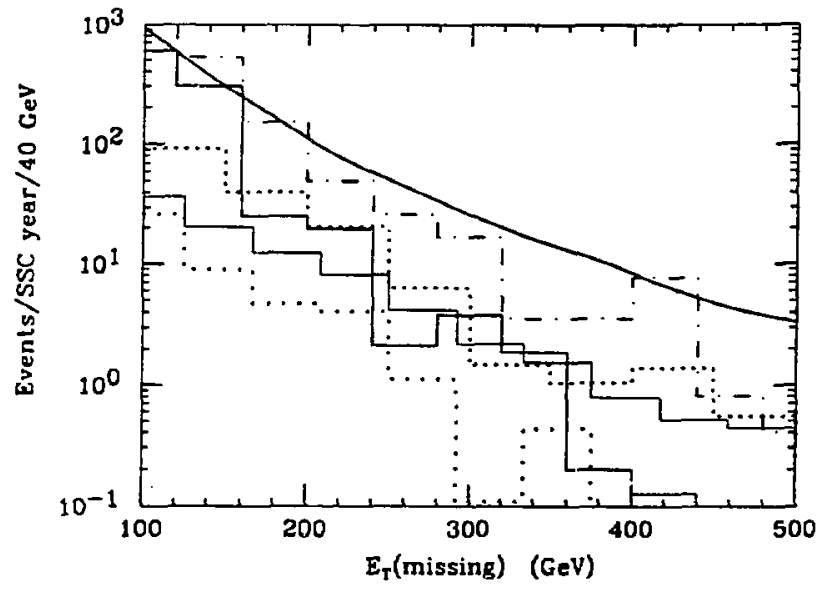

Figure 19: The distribution in missing $E_{T}$ showing various backgrounds to the search for a Higgs boson. The events are required to have a pair of leptons with invarjant mass $M_{Z} \pm 20 \mathrm{GeV}$ and $p_{t}>250 \mathrm{GeV}$. Each lepton is required to have $p_{z}>20 \mathrm{GeV}$. The background shown as a solid curve asises from $q \bar{q} \rightarrow Z Z$ (multiplied by 1.65 to account for the $g g \rightarrow Z Z$ process). The background (dot-dashed) arises from the final state $Z+j$ jets where the missing $E_{\mathrm{f}}$ is generated by calorimeter resolution or by losing energy out of the end of the detector. If events which contain a jet with $E_{T}>300 \mathrm{GeV}$ are vetoed, this background is reduced to the heavy solid histogram. The close-dotted bistogram arises from the final state $t \bar{t}$ where there is an $e^{+} e^{-}$(or $\mu^{+} \mu^{-}$) pair of mass $M_{Z} \pm 20 \mathrm{GeV}$ and the missing $E_{T}$ is due to neutrinos. The light-solid (wide-dotted) histogram arises from the $Z+t \bar{T}(Z+b \bar{b})$ final state. 


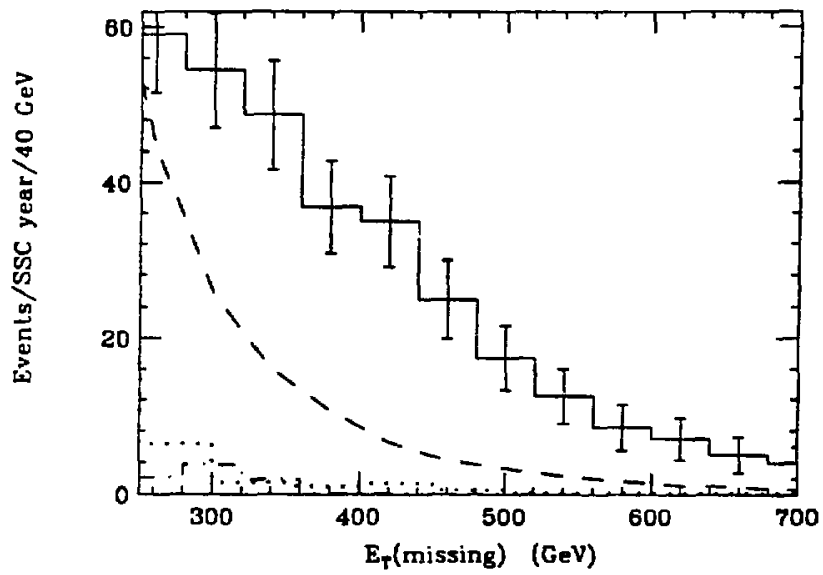

Figure 20: The distribution (solid histogram) in missing $E_{T}$ for the final state $Z\left(\rightarrow \mu^{+} \mu^{-}, e^{+} e^{-}\right)+$missing $E_{T}$ including the effect of a Higgs boson of mass $800 \mathrm{GeV}$ and the various backgrounds. The reconstructed $Z$ is required to have $p_{T}>250 \mathrm{GeV}$ and the events are rejected if they contain a jet with $E_{T}>300$ $\mathrm{GeV}$. For clarity, the figure shows the separate components of the background. The background shown as a dashed curve arises from $q \bar{q} \rightarrow Z Z$ (multiplied by 1.65 to account for the $g g \rightarrow Z Z$ process). The dot-dashed background arises from the final state $Z+j$ jets where the missing $E_{t}$ is generated by calorimeter resolution or by losing energy out of the end of the detector. The dotted background arises from the final state $t \bar{t}$ where there is an $e^{+} e^{-}$(or $\mu^{+} \mu^{-}$) pair of mass $M_{z} \pm 20 \mathrm{GeV}$ and the missing $E_{T}$ is due to neutrinos. 


\section{Searches at Higher Luminosity}

We have demonstrated that the searches for the Higgs boson are limited by the event rates and not by backgrounds. The search range can therefore be extended by increasing the integrated luminosity of the data sample.

The lower limit of our sensitivity to a Higgs decaying into the $Z Z^{*}$ final state cannot be extended very much by an increase in luminosity. There are two reasons for this. As can be seen from Fig. 2, the branching ratio to $Z Z^{\bullet}$ is falling rapidly as $M_{H}$ decreases, falling by a factor of three as $M_{H}$ varies between $125 \mathrm{GeV}$ and $115 \mathrm{GeV}$. The transverse momentum of the leptons is also decreasing as $M_{H}$ decreases, causing a reduction in our acceptance (we have assumed that we cannot control the backgrounds to leptons with $p_{\mathrm{t}}$ less than $10 \mathrm{GeV}$ ). We have initiated studies of the effect of pile-up at luminosities approaching $10^{34} \mathrm{~cm}^{-2} \mathrm{sec}^{-1}$, and preliminary results indicate that obtaining a rejection factor of ten against leptons from $b$ and lighter quarks appears feasible but challenging ir. this low $p_{t}$ region. We estimate that a run that accumulates $s 0$ integrated luminosity of $10^{41} \mathrm{~cm}^{-2}$ could be expected to be sensitive to Higgs masses above $\sim 120 \mathrm{GeV}$ in the $Z Z$ * channel. The $W+H \rightarrow e(\mu) \nu+\gamma \gamma$ channel described in Ref. [15] can extend this range down to about $80 \mathrm{GeV}$ for this integrated luminosity, but also requires similar rejections against non-isolated low $p_{t}$ leptons. Note that in the region where both $H \rightarrow Z Z^{*}$ and $H \rightarrow \gamma \gamma$ are detectable, it is possible to gain insight into the nature of the Higgs by measuring the relative branching ratios into the two modes.

At very large values of the Higgs mass, we are again limited by event rate. In the case of the $4 \ell$ channel, we are confident that the effect of pile-up will not significantly modify the backgrounds displayed ic Fig. 18 as the luminosity approaches $10^{34} \mathrm{~cm}^{-2} \mathrm{sec}^{-1}$. Preliminary studies of the muon resolution, assuming that the outer layer of the central tracker and the full muon system function normally, indicate that the resolution in the relevant $p_{t}$ range will deteriorate by less than a factor two (see Fig. 14 in the SDC LoI[21]). This change in resolution can be easily compensated by enlarging the window used for $Z$ selection to $\pm 20 \mathrm{GeV}$, with only small increases in the background. Figure 21 shows a simulation of a higher luminosity run, assuming the same resolutions and backgrounds as those shown in Fig. 18.

In the case of the $2 \ell 2 \nu$ final state, experiments at higher luminosity are likely to be affected by the need to modify or replace the calorimeter in the forward region. In order to estimate this effect we have recomputed the $Z+$ jets background assuming that the calorimeter does not extend beyond $|\eta|=4$, where the radiation dose for $10^{34} \mathrm{~cm}^{-2} \mathrm{sec}^{-1}$ is comparable to that at $\eta=5$ for $10^{33} \mathrm{~cm}^{-2} \mathrm{sec}^{-1}$. This loss of coverage will worsen 


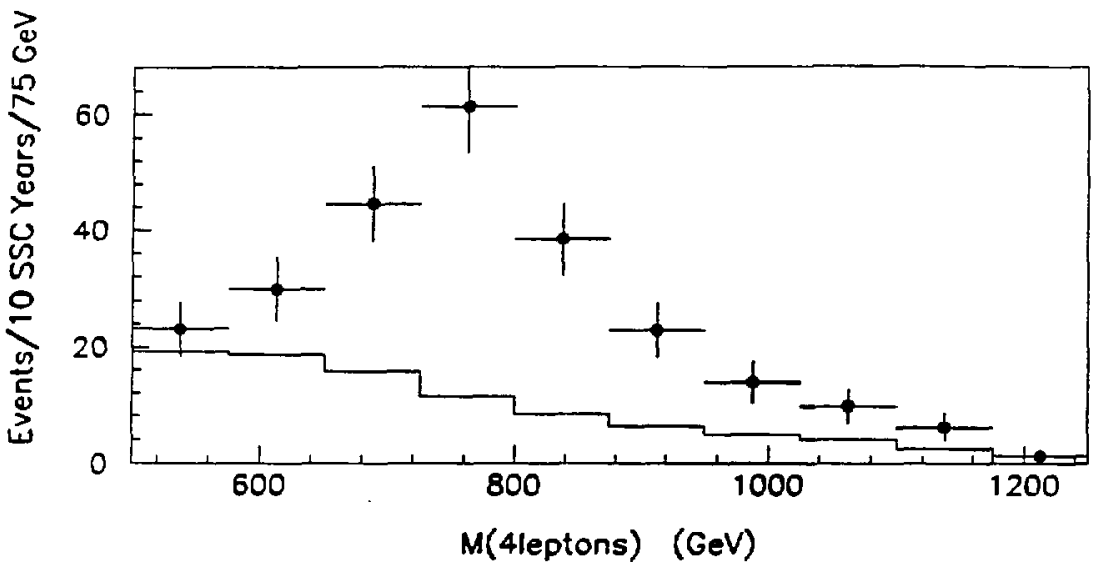

Figure 21: Same as Fig. 18, except that the integrated luminosity was taken to be 10 nominal SSC years.

the missing $E_{T}$ resolution of the detector. Nevertheless, it can be seen from Fig. 22 that we are still dominated by real (detector independent) backgrounds and that therefore this decay mode remains viable at higher luminosity. This conclusion is valid since the signal is confined to missing $E_{T}$ greater than $300 \mathrm{GeV}$. The smaller missing $E_{\tau}$ signal that would result from the decay of say a $300 \mathrm{GeV}$ Higgs would likely be compromised at higher luminosity. However, as we have seen, such a particle would have already been discovered before the luminosity was raised.

We have confidence that we could extract a signal from a Higgs boson of mass 800 G. . or more at a luminosity of $10^{34} \mathrm{~cm}^{-2} \mathrm{sec}^{-1}$. Hence we can extend the search into tne region where is the Weinberg-Salar. model becomes strongly coupled. We do not expect to be limited by the detector in our ability to extract a sigaal from such strongly interacting models. Rather the limitation will arise from intrinsic (detector independent) backgrounds [22]. 


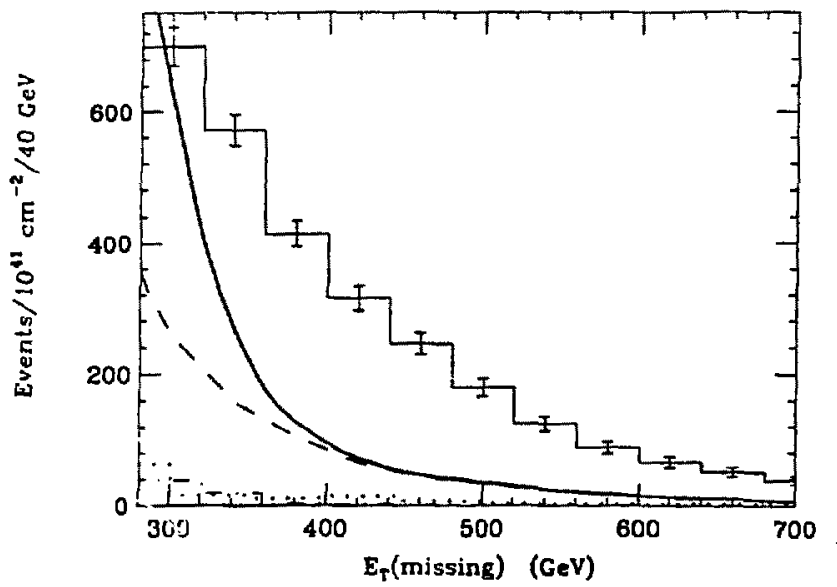

Figure 22: The distribution (solid histogram) in missing $E_{T}$ for the final state $Z\left(\rightarrow \mu^{+} \mu^{-}, e^{+} e^{-}\right)+$missing $E_{T}$ including the effect of a Higgs boson of mass $800 \mathrm{GeV}$ and the various backgrounds. We have assumed an integrated luminosity of $10^{41} \mathrm{~cm}^{-2}$ and that the calorimeter does not extend beyond $|\eta|=4$. The reconstructed $Z$ is required to bave $p_{T}>250 \mathrm{GeV}$ and the events are rejected if they contain a jet with $E_{T}>300 \mathrm{GeV}$. For clarity, the figure shows the separate components of the background. The background shown as a dashed curve arises from $q \vec{q} \rightarrow Z Z$ (multiplied by 1.65 to account for the $g g \rightarrow Z Z$ process). The background (dot-dashed) arises from the final state $Z+j$ jets where the missing $E_{T}$ is geverated by calorimeter resolution. The background (dotted) arises from the final state $t^{-}$where there is an $\mathrm{e}^{+} \mathrm{e}^{-}$(or $\mathrm{H}^{+} \mathrm{H}^{-}$) pair of mass $M_{Z} \pm 20 \mathrm{GeV}$ and the missing $E_{T}$ is due to Deutrinos. The solid curve is the sum of the above backgrounds plus that from the final state of $Z+$ jets where at least one jet escapes out of the end of the detector. 


\section{Conclusions}

We have demonstrated t'at the SDC detector, with its hermetic calorimetry and powerful tracking system, is capable of finding a Standard Model Higgs boson in the final states $e^{+} e^{-} e^{+} e^{-}, \mu^{+} \mu^{-} e^{+} e^{-}, \mu^{+} \mu^{-} \mu^{+} \mu^{-}, e^{+} e^{-\nu \bar{\nu}}$ and $\mu^{+} \mu^{-} \nu \bar{\nu}$ at the design luminosity of the SSC if its mass is in the range $125<M_{H}<800 \mathrm{GeV}$. Througbout this range, the significant backgrounds are those irreducible ones which produce real $Z Z$ or $Z Z^{*}$ final states which cannol be eliminated by improvements in detector resolution. The lower end of this range cannot be extended significantly by running at higher luminosity; another method is needed [15]. The higher end of the range can be extended by a run at a luminosity of $10^{34} \mathrm{~cm}^{-2} \mathrm{sec}^{-1}$.

\section{References}

[1] H.M. Georgi et al., Phys. Rev. Lett. 40:692 (1978).

[2] R.N. Cahn and S. Dawson, Phys. Lett. B 136:196 (1986);

S. Petcov and D.R.T. Jones, Phys. Lett. B 84:660 (1979).

(3) F. Abe et al. Phys. Rev. Lett. 64, 142 (1990)

[4] L. Amaldi et al. Phys. Rev. D36, 1985 (1987), P. Langacker Phys. Rev. Lett. 63, 1920 (1989), G. Costa, et al. Nucl. Phys. B297, 244 (1988)

[5] F. Abe et al, Phys. Rev. Lett. , (1989) (to appear).

[6] G. Arnison et al., Phys. Letl. 118B, 167 (1982).

[7] For more discussion see 1. Hinchlifte and M.D. Shapiro in Procedings of the 1988 Snowmass Summer Study Ed. R Donaldson.

[8] N. Sakai Phys. Rev. D22, 2220 (1980).

[9] H-U. Bengtsson and T. Sjostrand, Comp. Phys. Comm. 46 43, (1987).

[10] I. Hinchliffe, SDC-90-00100.

(11) T. Sjostrand, private communication of correct $\epsilon$ values to use.

[12] Y'. Sakai, SDC-90-00105.

[13] J. F. Gunion, G. Kane and J. Wudka Nucl. Phys. B299, 231 (1988) 
[14] J. J. van der Bij and E.W.N. Glover Phys. Lett. 206B, 701 (1988).

[15] M. Mangano, SDC-90-00113.

[16] R. W. Brown and K. O. Mikaelian, Phys. Rev. D19,922 (1979).

[17] E. W. N. Glover and J. J. van der Bij Nucl. Phys. B321, 561 (1989)

[18] R. N. Cahn et al, in Proc. of the 1987 Workshop on Detectors and Experimental Areas at the SSC, Ed. M. Gilchriese (World Scientific).

[19] T. Hessing and S. Behrends, "Inclusive Jet Spectrum $E_{t}$ Corrections and Resolution Unsmearing", CDF internal note 1132.

[20] R. M. Barnett, E. Carlson and I. Hinchliffe, SSC-SDE-29.

[21] Let ter of Intent submitted by the SDC collaboration, December 1990.

[22] V. Barger ef al. MAD/PH-556 (1990). 